\title{
Pulse propagation in gravity currents
}

\author{
P. A. Allen, ${ }^{1, \text { a) }}$ R. M. Dorrell, ${ }^{2}$ O. G. Harlen, ${ }^{3}$ R. E. Thomas, ${ }^{2}$ and W. D. McCaffrey ${ }^{4}$ \\ ${ }^{1)}$ School of Computing, Faculty of Engineering and Physical Sciences, University of Leeds, Leeds LS2 9JT, \\ $U K$ \\ 2) Energy and Environment Institute, University of Hull, Hull HU6 7RX, UK \\ ${ }^{3)}$ School of Mathematics, Faculty of Engineering and Physical Sciences, University of Leeds, Leeds LS2 9JT, \\ $U K$ \\ ${ }^{4)}$ School of Earth and Environment, Faculty of Environment, University of Leeds, Leeds LS2 9JT, \\ $U K$
}

(Dated: 10 December 2019)

\begin{abstract}
Real world gravity current flows rarely exist as a single discrete event, but are instead made up of multiple surges. This paper examines the propagation of surges as pulses in gravity currents. Using theoretical shallow-water modeling, we analyze the structure of pulsed flows created by the sequential release of two lock-boxes. The first release creates a gravity current, while the second creates a pulse that eventually propagates to the head of the first current. Two parameters determine the flow structure: the densimetric Froude number at the head of the current, $F r$, and a dimensionless time between releases, $t_{\mathrm{re}}$. The shallow-water model enables the flow behavior to be mapped in $\left(F r, t_{\mathrm{re}}\right)$ space. Pulse speed depends on three critical characteristic curves: two that derive from the first release and correspond to a wavelike disturbance which reflects between the head of the current and the back of the lock-box and a third that originates from the second release and represents the region of the flow affected by the finite supply of source material. Pulses have non-negative acceleration until they intersect the third characteristic, after which they decelerate. Variations in pulse speed affect energy transfer and dissipation. Critically for lahars, landslides and avalanches pulsed flows may change from erosional to depositional further affecting their dynamics. Gravity current hazard prediction models for such surge-prone flows may under-predict risk if they neglect internal flow dynamics.
\end{abstract}

\section{INTRODUCTION}

Gravity currents are flows driven by pressure gradients resulting from density differences. These may be the result of temperature, suspended sediment or salinity differences. Gravity currents form significant geophysical flows in atmospheric, terrestrial and subaqueous environments. Examples include, landslides, avalanches, turbidity currents, pyroclastic flows and lahars ${ }^{1}$. Pulses are a common feature in gravity currents and may result from flow instabilities, variable supply of dense material ${ }^{2}$, combining of flows from different sources, flow splitting and recombining ${ }^{3}$, or flow interactions with topography ${ }^{4}$. For example, failure mechanisms for landslides and similar events are varied ${ }^{5}$ and can result in pulsed flows: an initial failure of an embankment or dam can create a steep main scarp as the supporting material slides away, which, in turn, can lead to a further 'retrogressive' failure. The process may repeat creating a significantly larger event comprised of many smaller pulses. Surges and pulses internal to gravity currents can have a significant impact on the hazards associated and flow properties when compared to a single release of the same volume. This is particularly significant for compositional gravity currents where the variations in velocity affect the deposition or erosion that can occur ${ }^{6}$.

A pyroclastic flow generated by the 1997 eruption of the Soufriére hills volcano contain three distinct major flow surges over its 25 minute duration ${ }^{7}$. Deposits from the first two major surges partially filled the main drainage channel which the pyroclastic flow flowed along. This caused the third

\footnotetext{
a)scpaa@leeds.ac.uk
}

to overspill and travel into a region considered to be at low risk. The release dynamics of these gravity currents and potential evolution downstream in multiple surges impacts the dynamics of the flow. The deposits left by previous surges, and information they contain, can be destroyed by subsequent surges making flow dynamics difficult to identify. Further, run-out length, inundation zones and hazards are affected by the internal dynamics.

Turbidity currents sub-marine gravity currents in which the density difference is caused by suspended sediment and have significant economic impact; they can travel with head speeds as high as $19 \mathrm{~ms}^{-18}$ and cause significant damage to sea floor equipment, including pipelines, oil rig moorings ${ }^{9}$, and seafloor telecommunication cables ${ }^{10,11}$. Turbidity currents are a key mechanism of sediment distribution from shallow to deep marine environments through the oceans and lead to create of sedimentary rocks (turbidites) which are linked to hydrocarbon reservoirs ${ }^{12}$. Deposits of seismically generated turbidity currents at the Cascadia margin, Washington USA record multiple currents that combined downstream at as many as seven confluences ${ }^{13}$. The separation time between the flows can be negligible or of the order a few hours. Further, experimental modeling has demonstrated that the signature of individual turbidity currents can be destroyed after the different events interact ${ }^{14}$.

Gravity currents have been extensively studied by theoretical and experimental approaches, often based around the idealized lock-release or lock-exchange problem, where dense fluid is released by the rapid removal of a gate, providing a well-controlled initial condition. This method provides a suitable means to create repeatable, spatially and temporally varying, fixed-volume currents that allow meaningful comparison to theoretical models ${ }^{15-19}$. Recently, surge effects have 
been studied in the laboratory for turbulent saline flows using multiple lock-releases, in which a series of lock-boxes positioned behind the first were released at set times after the initial release ${ }^{14,20,21}$. The second current intruded into the first release and propagated towards the head of the current.

Dimensional arguments and simple integral models are able to predict the position of the head $x_{N}$ as a function of the initial conditions by assuming a dominant balance between buoyancy and either inertial or viscous forces ${ }^{17,22,23}$. By exploiting the large length $L$ and shallow depth $h$ of these flows, a first order asymptotic approximation in the aspect ratio $\delta=h / L$ followed by depth-averaging produces the hyperbolic shallowwater equations ${ }^{24}$. After an initial transience the flows generally enter a self-similar phase where the flow structure is independent of time and length scales. These similarity solutions capture some of the horizontal features of the flow. However, similarity solutions do not always exist. For example, in the axisymmetric spreading of a compositional gravity current, the tail of the current is self-similar but the head is time-dependent ${ }^{25}$.

The neglected vertical gradients in the shallow water model become significant at the head of the flow where turbulent drag and three-dimensional flow structures dissipate momentum. To capture this dissipation, shallow-water models are supplemented with an imposed dimensionless flow velocity at the head via a densimetric Froude number condition ${ }^{26}$. The densimetric Froude number is a dimensionless velocity of the current $F r=u / \sqrt{g^{\prime} h}$, where $g^{\prime}$ is the buoyancy adjusted gravity, $u$ is the streamwise velocity and $h$ is the flow depth, and is ratio between flow speed and infinitesimal long surface waves on the gravity current ${ }^{27}$. The buoyancy adjusted gravity expressed in terms of the current and ambient densities, $\rho_{c}$ and $\rho_{a}$, respectively, and gravitational acceleration $g$ is $g^{\prime}=g\left(\rho_{c}-\rho_{a}\right) / \rho_{c}$. Theoretical values of the Froude number can be determined through application of Bernoulli's theorem and a momentum balance of far upstream and far downstream of the head of the current in rectangular channels ${ }^{28,29}$ or by a vorticity balance without a dissipation assumption ${ }^{30}$ for Boussinesq flows. These differing approaches have been demonstrated to only differ by an assumption about the dissipation and can be reconciled within the same framework ${ }^{31}$. In addition, densimetric Froude number conditions have been calculated for a stratified ambient $t^{32}$ and non-rectangular cross-sections ${ }^{33}$. The densimetric Froude number condition can also be determined experimentally to be $1.2^{16}$. For non-Boussinesq flows, where the density difference between the current and the ambient is large, the Froude number can be large and even tend to infinity. This corresponds to zero depth at the front of the flow ${ }^{24}$.

Self-similar solutions of the lock-release problem provide a valuable tool for numerical model validation and enable development of these models to explore more complicated scenarios. In the majority of applications, numerical integration is required to obtain solutions. Direct numerical simulation (DNS) of the Navier-Stokes equations has also been conducted and demonstrates that depth-averaged models are able to capture the principal physics of gravity current dynamics with significantly reduced computational time $^{9}$. Shallow-water models can capture shocks (pulses) which appear as discontinuities in the solution. In reality, the viscosity of the flow smooths the discontinuity, but the shocks are still able to accurately capture the velocity of such disturbances $^{34}$. Bonnecaze, Huppert, and Lister ${ }^{34}$ employed a Lax-Wendroff finite-difference scheme to numerically integrate their shallow-water model that included sediment transport for a lock-release problem. The scheme accurately captured internal shocks which occurred behind the head downstream from the lock.

Hogg 27 employed the method of characteristics to solve the problem of a single-lock release flow. The shallow-water equations yield two families of characteristic curves along which the Riemann invariants $\alpha=u+\sqrt{g^{\prime} h}$ and $\beta=u-\sqrt{g^{\prime} h}$ are conserved. Hogg 27 showed that the structure of the solution depended qualitatively on the densimetric Froude number, Fr. For $F r \geq 2$, characteristics leaving the back of the head never reached the back of the lock-box and an internal shock formed for $F r>2$. For $F r<2$, a structured solution exists in which the characteristic $(x, t)$-space is split into regions where both, one or neither of the two characteristics variables are constant. Hogg 27 analytically determined the boundary between these regions and the solution when at least one of the characteristic variables is constant. These models have not been extended to the multiple release case.

The goal of this paper is to extend the lock-release problem for the shallow-water equations to a double-release case, where a second equally sized lock-box is released subsequently. The second release will create a shock that will propagate through towards the head current. A Lax-Wendroff finite-difference scheme based on the implementation of Bonnecaze, Huppert, and Lister ${ }^{34}$ is employed to solve the governing shallow-water equations. The characteristics are then computed from this solution afterwards, in order to describe the form of the solution in $(x, t)$ space. For a double-release problem with identical lock-boxes, there is an additional parameter as well as the densimetric Froude number: the dimensionless release time $t_{\mathrm{re}}^{*}=t_{\mathrm{re}} \sqrt{g^{\prime} h_{\text {lock }}} / l$, where $t_{\mathrm{re}}$ is the gate separation time, $h_{\text {lock }}$ is the lock depth and $l$ is the lock length. The work presented here explores the $\left(F r, t_{\mathrm{re}}\right)$ parameter space for $F r<2$. Simulations of the single-release case are compared to the analytical solution of $\operatorname{Hogg}^{27}$ for validation. The double-release simulations reveal a variety of distinct regions in the $\left(F r, t_{\text {re }}\right)$ parameter space with qualitatively different behavior in the shock velocity. For $t_{\mathrm{re}} \rightarrow \infty$, the two releases behave as two non-interacting events, whereas for $t_{\mathrm{re}} \leq 1$ the flow is effectively a single discrete event of twice the volume. However, the two events interact, affecting pulse propagation, for intermediate $t_{\mathrm{re}}$-values and a range of qualitatively different solutions are obtained. Regions are separated by whether or not they are affected by the amount of material in the second lock-box and the path through the single release solution structure. The velocity of the shock has implications for the dynamics of the pulsed gravity currents flows discussed earlier and the assessment of their hazards.

The paper is structured as follows: The shallow water model is presented for both the single- and double-release configurations in §II; Results from our numerical model and 
the parametric study are presented in §III; Implications are discussed in section $\S I V$; Finally, we conclude in $\S \mathrm{V}$.

\section{THEORETICAL MODELING}

Consider a constant volume gravity current propagating over a fixed horizontal rigid surface in two spatial dimensions $(x, z)$, where $x$ and $z$ are the horizontal and vertical coordinates, respectively, with the time from the first release defined as $t$, Fig. 1. The current has density $\rho_{c}$ and the deep ambient has density $\rho_{a}$. Thus, the buoyancy-adjusted gravity for the current may be expressed as $g^{\prime}=\left(\rho_{c}-\rho_{a}\right) g / \rho_{c}$, where $g=9.81 \mathrm{~ms}^{-2}$. In the lock-gate configuration, the flow quickly reaches a state where the height $h(x, t)$ is much smaller than the length of the current $x_{N}(t)$. Therefore, in considering the horizontal momentum of the flow we can average over the depth and assume purely hydrostatic pressure. Further, the flows are assumed inviscid (with no basal drag), entrainment is negligible, and the ambient is quiescent and infinitely deep. These assumptions allow us to apply the simplified depth-averaged shallow-water equations

$$
\begin{aligned}
\frac{\partial h}{\partial t}+\frac{\partial m}{\partial x} & =0, \\
\frac{\partial m}{\partial t}+\frac{\partial}{\partial x}\left(\frac{m^{2}}{h}+g^{\prime} \frac{h^{2}}{2}\right) & =0,
\end{aligned}
$$

35 , where

$$
m(x, t)=\int_{0}^{\infty} v(x, z, t) \mathrm{d} z \quad \text { and } \quad h(x, t)=\frac{\left(\int_{0}^{\infty} v(x, z, t) \mathrm{d} z\right)^{2}}{\int_{0}^{\infty} v(x, z, t)^{2} \mathrm{~d} z}
$$

are respectively the depth-integrated momentum per unit mass and the depth of the flow expressed in terms of the horizontal velocity $v(x, z, t)^{36}$. The shallow water equations are first order approximations in terms of the aspect ratio between the depth and length of the current $\delta$ and contain no source terms, i.e. drag and entrainment. However, at the head of the current $x=x_{N}(t)$ the dissipation is accounted for via a densimetric Froude number condition ${ }^{29}$. Further, a dynamic boundary condition is imposed at the head

$$
\frac{\mathrm{d} x_{N}}{\mathrm{~d} t} \equiv \dot{x}_{N}=\frac{m\left(x_{N}, t\right)}{h\left(x_{N}, t\right)}=\sqrt{g^{\prime} h\left(x_{N}, t\right)} F r
$$

where $\mathrm{Fr}$ is a constant and subscript $N$ denotes a value at the head. Both lock-boxes are assumed to be of the same length $l$ and filled to a depth of $h_{\text {lock}}$. Initially, no flux boundary conditions are imposed at the back of both lock-boxes $x=0, l$. After the second gate is released at $t=t_{\text {re }}$, the no flux condition at $x=l$ is removed. From the momentum equation (2), no flux is equivalent to $\partial h / \partial x=0$ and so

$$
\frac{\partial h}{\partial x}\left(x_{0}, t\right)=0 \text { for } \begin{cases}x_{0}=0, l & \text { if } t \leq t_{\mathrm{re}} \\ x_{0}=0 & \text { if } t>t_{\mathrm{re}}\end{cases}
$$

Similarly, the initial conditions, Fig. 1, are defined as

$$
h(x, 0)= \begin{cases}h_{\text {lock }} & \text { if } x \in[0,2 l] \\ 0 & \text { otherwise }\end{cases}
$$

The mass and momentum conservation equations (1) \& (2) are non-denationalized using $l$ as the horizontal length scale, $h_{\text {lock }}$ as the depth scale, $\sqrt{g^{\prime} h_{\text {lock }}^{3}}$ as the momentum scale and $l / \sqrt{g^{\prime} h_{\text {lock }}}$ as the convective time-scale :

$$
\begin{aligned}
\frac{\partial h^{*}}{\partial t^{*}}+\frac{\partial m^{*}}{\partial x^{*}} & =0, \\
\frac{\partial m^{*}}{\partial t^{*}}+\frac{\partial}{\partial x^{*}}\left(\frac{m^{* 2}}{h^{*}}+\frac{h^{* 2}}{2}\right) & =0,
\end{aligned}
$$

where $^{*}$ denotes a dimensionless variable. Similarly, the dimensionless boundary conditions (5) and (4) are:

$$
\begin{gathered}
\frac{\partial h^{*}}{\partial x^{*}}\left(x_{0}^{*}, t^{*}\right)=0 \text { for } \begin{cases}x_{0}^{*}=0,1 & \text { if } 0 \leq t^{*} \leq t_{\mathrm{re}}^{*}, \\
x_{0}^{*}=0 & \text { if } t^{*}>t_{\mathrm{re}}^{*}\end{cases} \\
\frac{\mathrm{d} x_{N}^{*}}{\mathrm{~d} t^{*}}=\frac{m_{N}^{*}}{h_{N}^{*}}=F r \sqrt{h_{N}^{*}}
\end{gathered}
$$

and initial conditions (6) become

$$
h^{*}\left(x^{*}, t^{*}\right)= \begin{cases}1 & \text { if } x^{*} \in[0,2] \\ 0 & \text { otherwise }\end{cases}
$$

\section{A. Analysis of characteristics: single release case}

This section will discuss the behavior of the flow for $t<t_{\mathrm{re}}$, which is equivalent to the single release solution of $\mathrm{Hogg}^{27}$. From this point the ${ }^{*}$ are neglected from the dimensionless variables for brevity, unless stated otherwise. The system of equations (7-8) can be transformed into its characteristic form ${ }^{24}$ by changing to characteristic variables

$$
\alpha=u+2 c, \quad \beta=u-2 c,
$$

where $u=m^{*} / h^{*}$ and $c=\sqrt{h^{*}}$ to obtain

$$
\begin{array}{lll}
\frac{\mathrm{d} \alpha}{\mathrm{d} t}=0 & \text { on } & \frac{\mathrm{d} x}{\mathrm{~d} t}=u+c \\
\frac{\mathrm{d} \beta}{\mathrm{d} t}=0 & \text { on } & \frac{\mathrm{d} x}{\mathrm{~d} t}=u-c .
\end{array}
$$

Thus, $\alpha$ and $\beta$, the Riemann invariants, are constant along characteristics curves with gradients in $(x, t)$-space of $u+c$ and $u-c$, respectively. The gradients $u \pm c$ are the eigenvalues of the system of equations (7) \& (8) and, provided the flow depth is non-zero, are real and distinct everywhere. Thus, the system is hyperbolic and the method of characteristics may be applied. The values of the characteristic variables can be determined from boundary or initial conditions that the characteristics pass through. 

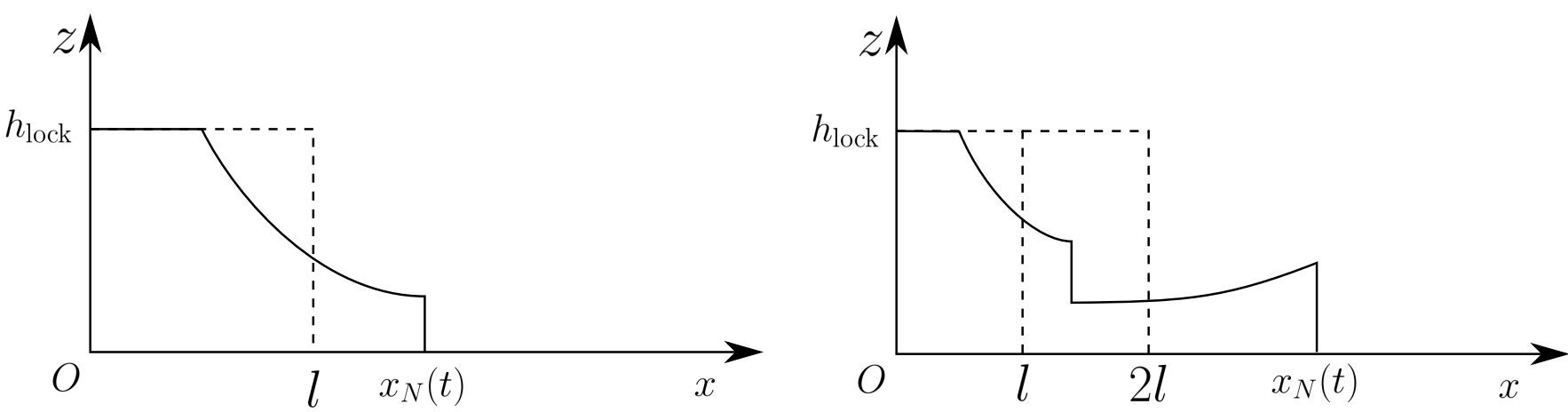

FIG. 1: Configuration of the single (left) and double (right) lock-release problems. Initial conditions (- -) and current depth, $h(x, t),(-)$ displayed.

Initially, $\alpha=2$ and $\beta=-2$ on all positive and negative characteristics that originate from $0<x<2, t=0$, see Fig. 2 . Thus, with the imposed densimetric Froude number condition $u_{N}=F r c_{N}$, positive characteristics collide with the head at a finite time provided $\mathrm{Fr} \nrightarrow \rightarrow \infty$ (in the limit $\mathrm{Fr} \rightarrow \infty$, the head corresponds to the leading characteristic). While positive characteristics arrive at the head with $\alpha=2$, the velocity and wave speed at the head are constant and take value

$$
u_{N}=F r c_{N} \quad \text { and } \quad c_{N}=\frac{2}{F r+2} .
$$

Thus, negative characteristics emanating from the back of head have constant

$$
\beta=\beta_{m} \equiv 2(F r-2) /(F r+2) \geq-2 .
$$

An expansion fan of negative characteristics emanates from $(2,0)$ and connects the two regions where $\beta$ is constant, see Fig. $2 b$. These negative characteristics correspond to straight lines through the origin and satisfy

$$
x=1+(4+6 \beta)(2-\beta)^{-\frac{3}{2} t} \quad \text { for } \quad \beta \in\left[-2, \beta_{m}\right] .
$$

The negative characteristics with $\beta=\beta_{m}$ and $\beta=-2$, and their subsequent reflections are denoted $x_{\text {fan }}(t)$ and $x_{\text {ref }}(t)$, respectively, and form the boundaries between uniform (U) and simple ( $\mathrm{S}$ ) wave regions in which either $\beta$ is constant or $\beta$ varies. The fastest backwards traveling negative characteristic, $x_{\text {ref }}(t)$, originates from the initial release at $x=2$ and represents the furthest part of the lock that is affected by the initial release. For clarity, the curves $x_{\text {ref }}$ and $x_{\text {fan }}$ represent different characteristics with different values of the conserved quantities after each reflection. They alternate between positive and negative characteristics between each branch. All positive characteristics intersecting the first branch of $x_{\text {ref }}$ arrive from unperturbed fluid and so have $\alpha=2$, yielding $\mathrm{d} x / \mathrm{d} t=-1$ and thus $x_{\text {ref }}(1)=1$. For $t>1$, the positive characteristics emanating from $t>1$ have $\alpha<2$, because $h<1$ and $u=0$. The last characteristic with $\alpha=2$ signifies the boundary of the region of the flow that is affected by the finite length of the first lockbox. For $t>1, x_{\text {ref }}$ defines the last characteristic with $\alpha=2$. The curves $x_{\text {ref }}$ and $x_{\text {fan }}$ collide at $t=(2+F r)^{3 / 2} / \sqrt{8}$. Beyond this point, $x_{\mathrm{fan}}$ enters a region of varying $\alpha$ and therefore has a non-constant gradient. In contrast, $x_{\text {ref }}$ enters a region of constant $\beta$ and thus has constant gradient until reaching the head. Hogg ${ }^{27}$ gives the $x_{\text {ref }}$ characteristic between the back wall and the head as

$$
x_{\mathrm{ref}}(t)= \begin{cases}2+2 t-3 t^{\frac{1}{3}} & \text { if } \quad t \in\left[1, \frac{(2+F r)^{\frac{3}{2}}}{\sqrt{8}}\right], \\ 2-\sqrt{2(2+F r)}+2 \frac{F r+1}{F r+2} t & \text { if } \quad t \in\left[\frac{(2+F r)^{\frac{3}{2}}}{\sqrt{8}}, \frac{(2+F r)^{\frac{3}{2}}}{\sqrt{2}}\right] .\end{cases}
$$

At times beyond this characteristic, complex (C) or simple (S) wave regions exist, where both or one characteristic varies in time, respectively. The lines $x_{\text {fan }}$ and $x_{\text {ref }}$ are continually defined through reflections as, respectively, the first or last characteristic upon which either $\alpha$ or $\beta$ are locally constant. For $F r<2$ they reflect between the head and the rear lock-box and divide the solution space into uniform (U), simple (S) and complex (C) wave regions, Fig. 3. Constant negative char- acteristics in regions $\mathrm{U}_{1}, \mathrm{~S}_{2}$ and $\mathrm{U}_{2}$ have $\beta=\beta_{m}$ (16) and thus, at the back of the first lock-box where, from the boundary condition (9), $u=0$ and hence $\alpha=-\beta=2 c=-\beta_{m}$, new positive characteristics in the regions $\mathrm{U}_{2}, \mathrm{~S}_{3}$ and $\mathrm{U}_{3}$ take the value $\alpha=-\beta_{m}$.

The densimetric Froude number condition (10) implies negative characteristics have $\beta=\beta_{m}(F r-2) /(F r+2)$ when positive characteristics with the constant value $\alpha=-\beta_{m}$ arrive at 


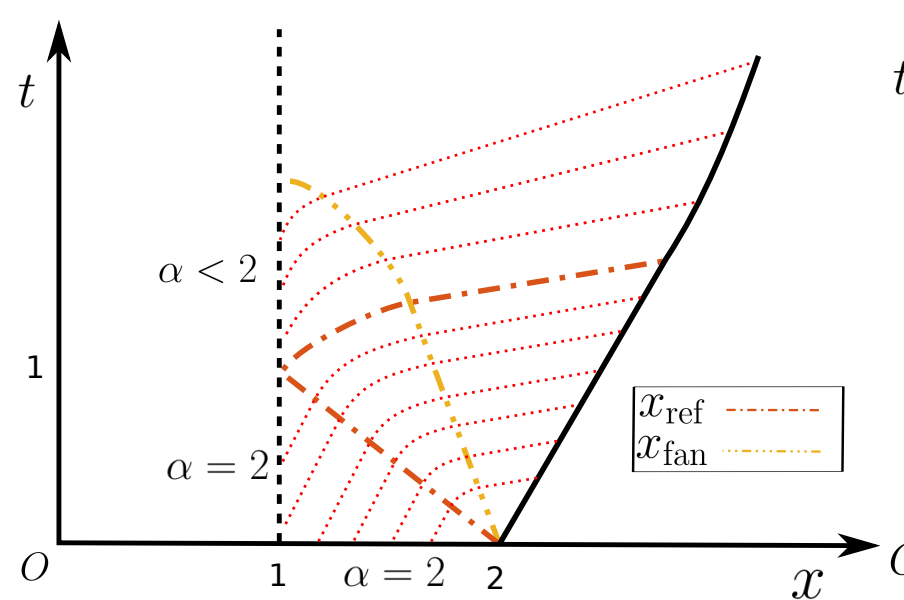

(a)

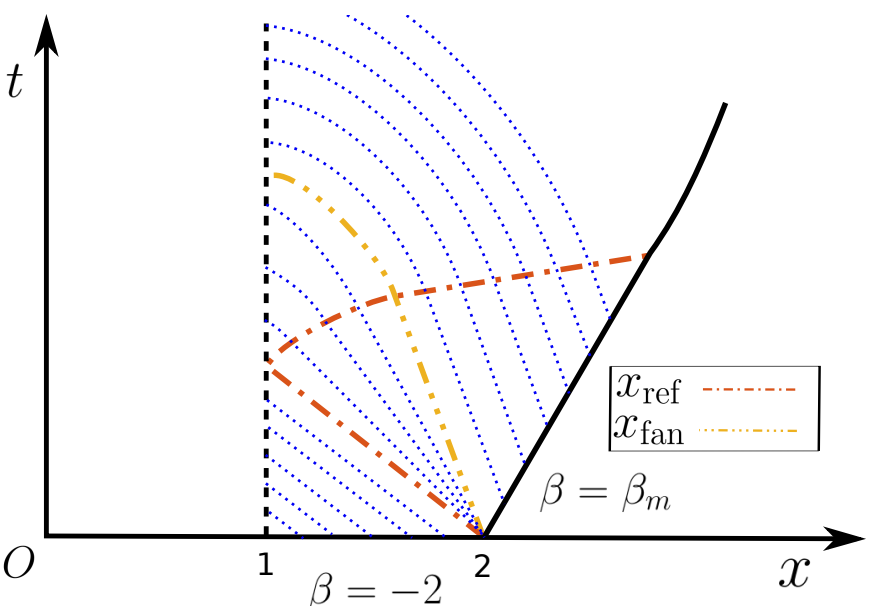

(b)

FIG. 2: Characteristic diagrams shortly after the first release for a densimetric Froude number $F r$ less than 1 .For $1<F r<2$, $x_{\text {ref }}$ has positive gradient until intersecting $x_{\text {fan }}$ The head of the current $x_{N}(-)$ moves at constant speed until $x_{\text {ref }}(\cdot-)$ collides with it. Positive (a) and negative (b) characteristics $(\cdots)$ are displayed for a small range of starting values. The boundary between the expansion fan and the constant region behind the head is $x_{\mathrm{fan}}(\cdots-)$. Online version in color.

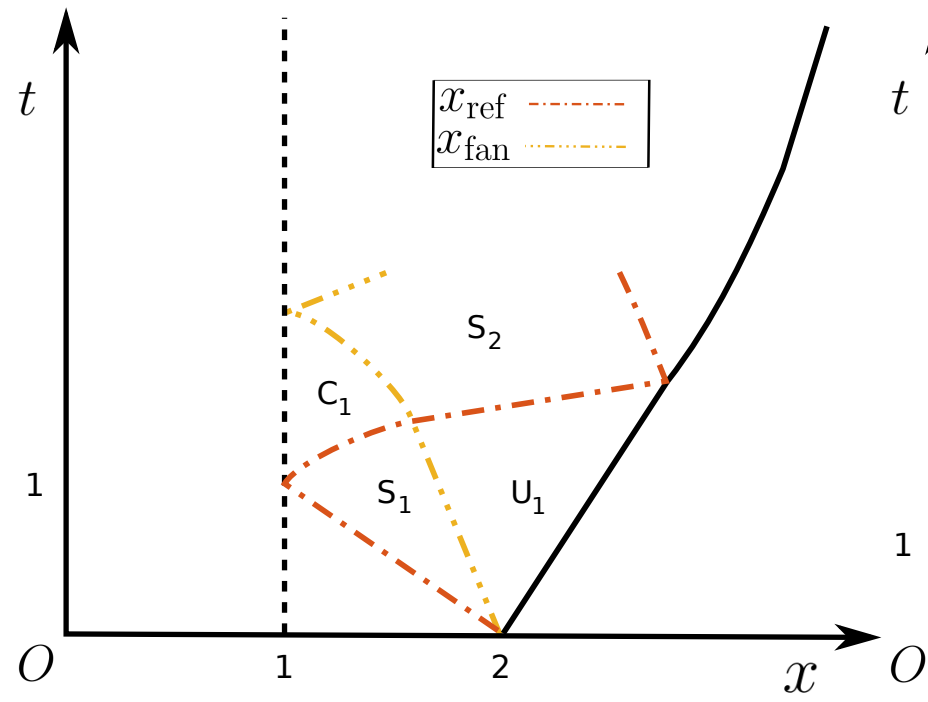

(a)

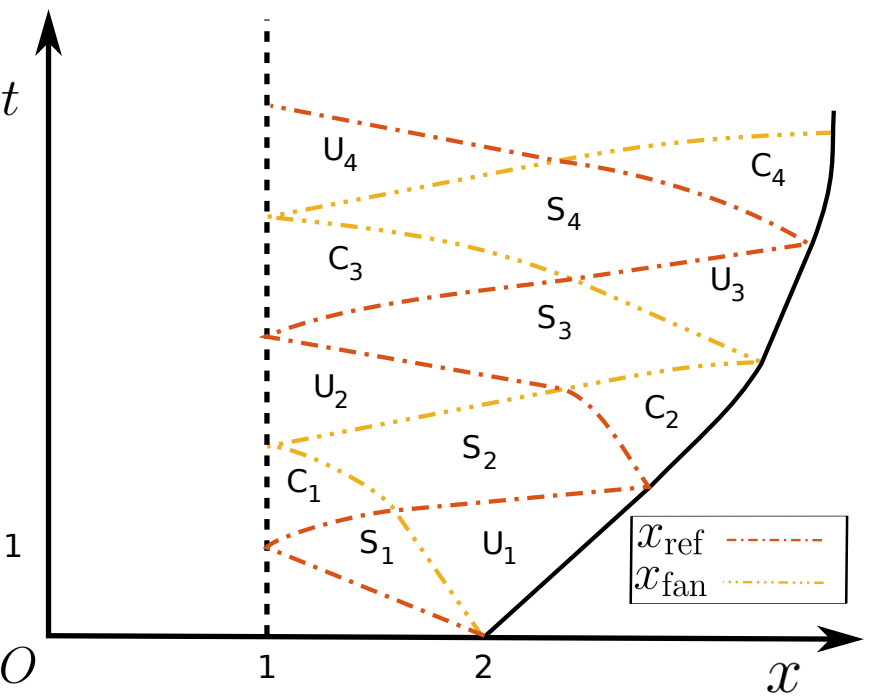

(b)

FIG. 3: Characteristic diagram highlighting the two curves $x_{\text {ref }}(\cdot-)$ and $x_{\text {fan }}(\cdots-)$ and how the solution is partitioned into uniform (U), simple (S) and complex (C) regions shortly after the release (a) and at a later time (b). Online version in color.

the head. This holds in regions $\mathrm{U}_{3}, \mathrm{~S}_{4}$ and $\mathrm{U}_{4}$. These boundary conditions continually create regions in the flow where the characteristics variables $\alpha$ or $\beta$ are constant. The values of $\alpha$ or $\beta$ may be calculated in any region in which they are constant $^{27}$. For $n \in \mathbb{N}$ regions $\mathrm{U}_{2 n}, \mathrm{~S}_{2 n+1}$ and $\mathrm{U}_{2 n+1}$ have

$$
\alpha=2\left[\frac{2-F r}{2+F r}\right]^{n} \equiv 2 \lambda^{n}
$$

because of the no flux boundary condition (9), while in regions
$\mathrm{U}_{2 n+1}, \mathrm{~S}_{2 n+2}$ and $\mathrm{U}_{2 n+2}$

$$
\beta=-2\left[\frac{2-F r}{2+F r}\right]^{n+1}=-2 \lambda^{n+1}
$$

because of the densimetric Froude number condition (10). As $n \rightarrow \infty, \alpha \rightarrow 0$, and $\beta \rightarrow 0$, and thus $u \rightarrow 0$ and $c \rightarrow 0$. When $F r \sim 0, \lambda \sim 1$ and therefore the flows will interact for a large number of reflections and hence longer release times. For $F r \sim 2$ there is minimal interaction between the events. Critically, $x_{\text {ref }}$ and $x_{\text {fan }}$ partition the single-release solution into three distinct regions for any fixed $t$ where the behavior of $\alpha$ 
and $\beta$ are qualitatively different.

\section{B. Extension to double release}

From the structure described in section II A we can determine the nature of the solution when the second gate is released at $t=t_{\mathrm{re}}$. If $t_{\mathrm{re}} \leq 1$ then trivially the solution behaves identically to a single-release of lock-box length 2 , because the backwards traveling disturbance has insufficient time to reach $x=1$. For $t_{\mathrm{re}}>1$, a shock is created where positive characteristics in $x<1$, having $\alpha=2$, collide with positive characteristics from $x \geq 1$ having $\alpha<2$. Depending on the release time, $t_{\mathrm{re}}$, the shock is released into a region of constant depth (uniform region) or varying depth and ve- locity (complex region) and so the relative position of the two curves, $x_{\text {ref }}$ and $x_{\text {fan }}$, determine the initial motion of the shock. Imposing that mass and momentum fluxes are conserved across the shock, the shock velocity $\dot{x}_{\mathrm{s}}$ can be obtained from the Rankine-Hugoniot conditions of the shallow water equations, because they are in a conservative form. In terms of the characteristic variables ahead of and behind the shock, $\alpha^{+}, \beta^{+}, \alpha^{-}, \beta^{-}$, respectively, the shock velocity $\dot{x}_{s}$ is

$$
\dot{x}_{\mathrm{s}}=\frac{1}{2} \frac{\left(\alpha^{+}+\beta^{+}\right)\left(\alpha^{+}-\beta^{+}\right)^{2}-\left(\alpha^{-}+\beta^{-}\right)\left(\alpha^{-}-\beta^{-}\right)^{2}}{\left(\alpha^{+}-\beta^{+}\right)^{2}-\left(\alpha^{-}-\beta^{-}\right)^{2}},
$$

from mass conservation (7) and

$$
\dot{x}_{\mathrm{s}}=\frac{1}{16} \frac{8\left(\alpha^{+}-\beta^{+}\right)^{2}\left(\alpha^{+}+\beta^{+}\right)^{2}-8\left(\alpha^{-}-\beta^{-}\right)^{2}\left(\alpha^{-}+\beta^{-}\right)^{2}+\left(\alpha^{+}-\beta^{+}\right)^{4}-\left(\alpha^{-}-\beta^{-}\right)^{4}}{\left(\alpha^{+}+\beta^{+}\right)\left(\alpha^{+}-\beta^{+}\right)^{2}-\left(\alpha^{-}+\beta^{-}\right)\left(\alpha^{-}-\beta^{-}\right)^{2}}
$$

from momentum conservation (8). Initially $\alpha^{-}=2$ as the positive characteristics come from unperturbed fluid. Shocks that propagate into a uniform region have both $\alpha^{+}$and $\beta^{+}$ constant and thus, by the Rankine-Hugoniot conditions (21) \& (22), both $\dot{x}_{\mathrm{s}}$ and $\beta^{-}$are also constant. In simple and complex regions, the shock will accelerate or decelerate and values of $\beta^{-}$will vary.

In uniform regions adjacent to $x=1$, the boundary condition $u(1)=0$ (9) implies that $u(x)=0$ throughout the uniform region. Thus, and whilst $\alpha^{-}=2$, the problem replicates the wet dam break ${ }^{24}$ and the shock velocity can be calculated explicitly throughout the uniform region. The RankineHugoniot conditions (21) \& (22) provide an implicit relation for the constant shock velocity, $\dot{x}_{\mathrm{s}}$, for uniform regions adjacent to the head. For a shock of positive velocity, $\dot{x}_{\mathrm{s}}$, causality implies that positive characteristics cannot be emitted by the shock. Thus, the shock represents the furthest point in the domain that has been affected by the release of the second gate.

Similar to the line $x_{\mathrm{ref}}(t)$, an additional line $x_{\mathrm{fin}}(t)$ is introduced for the second release. The first branch tracks the backwards propagating disturbance of the second release, i.e. the fastest negative characteristic from $\left(1, t_{\mathrm{re}}\right)$. On this characteristic $\beta=-2$, and positive characteristics intersecting it arrive from unperturbed fluid and therefore $\alpha=2$. Thus

$$
\frac{\mathrm{d} x_{\mathrm{fin}}}{\mathrm{d} t}=-c=-1
$$

and so

$$
x_{\mathrm{fin}}(t)=1-\left(t-t_{\mathrm{re}}\right) \quad \text { for } \quad t \in\left[t_{\mathrm{re}}, t_{\mathrm{re}}+1\right] .
$$

At $t=t_{\mathrm{re}}+1$, the fluid at the back of the second lock starts to be affected by the gate release and beyond this time $\alpha<2$ at $x=0$. The last $\alpha=2$ characteristic leaves $x=0$ at $t=$ $t_{\mathrm{re}}+1$, which is denoted as the continuation of the line $x_{\mathrm{fin}}$. The second branch of the curve $x_{\text {fin }}$ defines the part of the solution affected by the finite length of the second lock-box. If this characteristic intersects the shock, then $\alpha^{-}<2$ thereafter.

Both $\beta^{-}$and the shock velocity $\dot{x}_{\mathrm{s}}$ are constant when the shock propagates through a uniform region. Thus, for a shock propagating in a uniform region, a region of constant $\beta$ is created, which in turn creates another region of constant $\alpha<2$ upon reaching the back of the lock-box. The structure of the characteristic space is displayed for a shock released into a uniform region, Fig. 4, and a complex region, Fig. 5. For shocks released into either uniform or complex regions negative characteristics will have gradients greater than -1 (14) and another expansion fan of negative characteristics must exist at $\left(1, t_{\mathrm{re}}\right)$. A shock released into a uniform region will initially travel at a constant speed. Further, the flow depth and velocity will be constant either side of the shock. This will hold until the shock intersects one of the three curves $x_{\text {ref }}, x_{\text {fan }}$, or $x_{\text {fin }}$, with each possibility leading to a different structure behind the shock. The example drawn in Fig. 4 has the shock intersecting $x_{\text {ref }}$ initially. For complex shocks, Fig. 5, the shock speed accelerates from the outset, because the values of $\alpha^{+}$and $\beta^{+}$are decreasing. The shock may exhibit a region of constant velocity, but only once it has intersected both $x_{\text {ref }}$ and $x_{\text {fan }}$. The three curves $x_{\text {ref }}, x_{\text {fan }}$ and $x_{\text {fin }}$ bound critical regions where characteristic variables $\alpha$ and $\beta$ change from either being constant or varying. The single release solution space is partitioned by $x_{\text {ref }}$ and $x_{\text {fan }}$ into regions with varying or constant $\alpha$ and $\beta$. Therefore, their position relative to the shock when it is released influences the initial shock velocity. The dynamics of the shock change when it collides with $x_{\text {ref }}$ or $x_{\text {fan }}$, which in turn affects the negative characteristics behind it. Further, when $x_{\text {fin }}$ intersects the shock, an additional change in dynamics occurs. The order in which these effects occur creates a range of different shock velocities.

The shallow-water equations (7) \& (8) coupled with boundary (9) \& (10) and initial (11) conditions are solved using the 


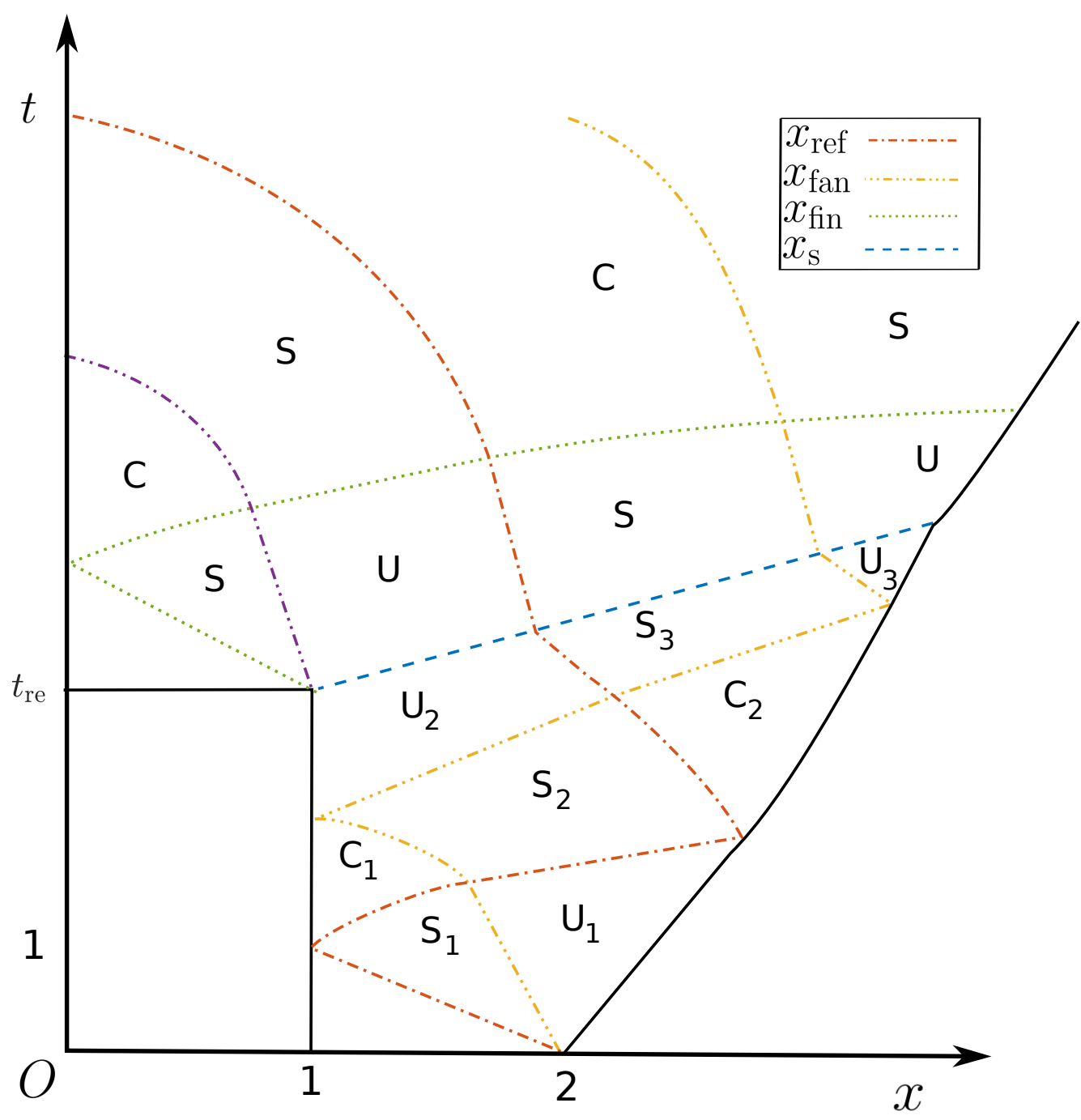

FIG. 4: Schematic showing an example structure of the characteristic space for a shock released into the $\mathrm{U}_{2}$ region. Ahead of the shock, the regions are bounded by the two lines $x_{\text {ref }}(\cdot-)$ and $x_{\text {fan }}(\cdots-)$, the head of the current $x_{N}(-)$ and the shock $x_{s}(--)$. At the point of release, an additional expansion fan is bounded between $x_{\mathrm{fin}}(\cdots)$ and the slowest backwards traveling characteristic from the second release $(\cdot-)$. Online version in color.

method of Bonnecaze, Huppert, and Lister ${ }^{34}$. For stability the initial depth, $h_{N}$, and velocity, $u_{N}$, at the head were set to slumping phase values;

$$
u_{N}=\frac{2 F r}{2+F r} \quad \text { and } \quad h_{N}=\left(\frac{2}{F r+2}\right)^{2} .
$$

Further, the shock initiates at $(x, t)=\left(1, t_{\mathrm{re}}\right)$, where $u=0$, and thus the positive and negative characteristics ahead of the shock takes values

$$
\alpha^{+}=2 \sqrt{h\left(1, t_{\mathrm{re}}\right)} \quad \text { and } \quad \beta^{+}=-2 \sqrt{h\left(1, t_{\mathrm{re}}\right)} .
$$

Together with $\alpha^{-}=2$, the Rankine-Hugoniot conditions (21) $\&(22)$ were used to determine the initial shock velocity and depth, $\dot{x}_{S}\left(t_{\mathrm{re}}\right)$, and these were imposed at the node coinciding with the shock for the first time step after release.

The shallow water equations are remapped to the unit interval using the change of variables $\left.(\zeta, \tau)=x / x_{N}(t), t\right)$. This removes the moving boundary condition simplifying the application of the Froude number condition. For full details see appendix A of Bonnecaze, Huppert, and Lister ${ }^{34}$. The transformed equations are solved using a Lax-Wendroff finite different scheme. Numerical integration with a upwind finitedifference scheme is used to determine the positive characteristic that arrives at the head, $\zeta=1$, at the next time step and provide, together with the Froude number condition, a second equation for $u$ and $c$ at the head. This enabled us to determine suitable values used as boundary conditions at the head for the next time step. Model validation is performed by comparing the numerical solution at $t=1$ and the curve $x_{\text {ref }}(t)$ with the exact solution given by Hogg 27 . This was chosen, because of the significance of the three lines $x_{\text {ref }}(t), x_{\text {fan }}(t)$ and $x_{\text {fin }}(t)$ to assess the ability of the simulations to capture the distinct regions where behavior changes. This validation is presented in the Appendix 




FIG. 5: Schematic showing an example structure of the characteristic space for a shock released into a $\mathrm{C}_{3}$ region. Ahead of the shock, the regions are bounded by the two lines $x_{\text {ref }}(\cdot-)$ and $x_{\text {fan }}(\cdots-)$, the head of the current $x_{N}(-)$ and the shock $x_{s}(--)$. At the point of release, an additional expansion fan is bounded between $x_{\mathrm{fin}}(\cdots)$ and the slowest backwards traveling characteristic from the second release $(\cdots-)$. Online version in color.

\section{RESULTS AND DISCUSSION}

In this section, the range of possible solutions from the shallow-water model are classified in terms of qualitative differences in the shock velocity throughout its motion towards the head of the current. The generic structure of the $\left(F r, t_{\mathrm{re}}\right)$ phase space is presented from a parametric study, and the solution types are presented.

\section{A. Shock Evolution}

The numerical solutions are first distinguished by the region the shock is released within, $\mathrm{C}_{1}, \mathrm{U}_{2}, \mathrm{C}_{3}$ etc. If the shock at $x_{\mathrm{s}}(t)$ is released into a uniform region, the shock velocity remains constant until it enters the simple wave region. If the shock is released into a complex region, its velocity varies from the outset. The characteristics that bound the region of varying or constant $\alpha$ and $\beta, x_{\text {fan }}(t)$ and $x_{\text {ref }}(t)$, are bounded by the flow front and must collide with the shock before it reaches the front. The intersection times, $t_{\text {fan }}$ and $t_{\text {ref }}$, i.e. $x_{\text {fan }}\left(t_{\text {fan }}\right)=x_{\mathrm{s}}\left(t_{\text {fan }}\right)$ and $x_{\text {ref }}\left(t_{\text {ref }}\right)=x_{\mathrm{s}}\left(t_{\text {ref }}\right)$, signify changes in behavior in front of the shock.

If the shock is released into a complex region, three distinct paths through $(x, t)$-space may occur: i) The shock first intersects $x_{\text {fan }}$ and enters a simple wave region in which $\beta$ is constant. The shock then intersects $x_{\text {ref }}$ after it has reflected off the front, entering another complex region; ii) The shock intersects $x_{\text {fan }}$ first, but intersects $x_{\text {ref }}$ before it has reflected off the head. Thus, the shock enters a uniform region until colliding with the head; iii) The shock intersects $x_{\text {ref }}$, entering a simple region within which $\alpha$ is constant. It then intersects 
$x_{\text {fan }}$, entering a uniform region. The three possibilities for $\mathrm{C}_{1}$ are displayed in Fig. 6a. A similar distinction is drawn for the uniform cases, for example $\mathrm{U}_{2}$ in Fig. $6 \mathrm{~b}$ : i) $\beta$ varies within the simple wave region before becoming constant again; ii) $\beta$ starts varying first followed by $\alpha$; iii) $\alpha$ starts varying followed by $\beta$. Further, if the shock is affected by the finite length of the domain lock-box, i.e. $x_{\text {fin }}$ intersects with the shock before it reaches the head of the flow, then this is equivalent to $\alpha^{-}<2$ beyond this point. The first time the shock and $x_{\text {fin }}$ coincide is defined as $t_{\text {fin }}$, such that $x_{\text {fin }}\left(t_{\text {fin }}\right)=x_{s}\left(t_{\text {fin }}\right)$. If the shock is affected by the finite length of the lock-box, the case is labeled ' $\mathrm{F}$ ', otherwise ' $\mathrm{N}$ '. For example $\mathrm{U}_{2} \mathrm{Fi}$ is a shock that is released into the $U_{2}$ region of type $i$ that is affected by the finite length of the lock-box.

Numerical solutions for the $\mathrm{U}_{2} \mathrm{~N}$ shocks are displayed in Fig. 7. For case i, Fig. 7a\&b, $\beta$ varies upon entering the $S_{3}$ region and the shock accelerates at approximately a constant rate until entering $\mathrm{U}_{3}$, where it returns to a constant but higher velocity until colliding with the head of the current. In contrast the shock velocity increases throughout in cases ii, Fig. $7 \mathrm{c} \& d$, and iii, Fig. 7e\&f, as first one characteristic starts varying and then the other ( $\beta$ first for case ii and $\alpha$ first for case iii). The shock velocity at $t_{\text {fin }}$ is largest for case iii, with case $\mathrm{i}$ being the slowest.

For larger densimetric Froude numbers, the shock has further to travel before reaching the front and therefore is more likely to be affected by the finite length of the lock-box. The $\mathrm{U}_{2} \mathrm{~F}$ shocks are qualitatively similar to the $\mathrm{U}_{2} \mathrm{~N}$ cases, Fig. 8 , until $x_{\text {fin }}$ collides with the shock at $t=t_{\text {fin }}$, after which the velocity decreases and the maximum shock velocity occurs before the head. Case $\mathrm{U}_{2} \mathrm{Fi}$ does not exist. i.e., the shock can only be affected by the finite length of the lock-box if it enters a region where $\alpha$ is varying. Further, our simulations reveal that this case $\mathrm{U}_{4} \mathrm{Fi}$ does not exist. Shocks released into complex regions immediately increase in velocity as both $\alpha^{+}$is decreasing and $\beta^{+}$is increasing. Example $\mathrm{C}_{3} \mathrm{~N}$ cases are presented in Fig. 9. As expected, the acceleration of the shock decreases after $t_{\text {fan }}$ for case $\mathrm{i}$, because $\beta$ takes a constant value until $t_{\text {ref }}$, after which $\beta$ starts decreasing again and the acceleration of the shock increases. For cases ii and iii, the acceleration of the shock decreases when it enters the $S_{3}$ region before becoming zero when entering $\mathrm{U}_{3}$ until reaching the head of the current. For $\mathrm{C}_{3}$ and subsequent complex and uniform cases, the shock may feel the affect of the finite length of the lock-box before colliding with $x_{\text {ref }}$ or $x_{\text {fan }}$. The acceleration of the shock still decreases after it is affected by the finite length of the domain, but the range of possibilities becomes more complex, Fig. 10.

\section{B. Classification of Solutions}

A large number of numerical simulations were run in the range of values $\left(F r, t_{\text {re }}\right) \in[0,2) \times(1,21]$ and from the three curves, $x_{\text {ref }}, x_{\text {fan }} \& x_{\text {fin }}$, and the shock, $x_{S}$, the corresponding case was determined. For each $F r$, the boundaries between regions were chosen at $t_{\mathrm{re}}$ values between the simulations of different cases. In an iterative process, further simulations were



(a)



(b)

FIG. 6: Schematic showing examples of the three characteristic diagrams for the three distinct paths $\mathrm{C}_{1}$ (a) and $\mathrm{U}_{2}$ (b) shocks, $x_{s}$, (- -) can take through the single release solution, i,ii and iii. The shock intercepts $x_{\text {ref }}$ and $x_{\text {fan }}(\cdots-)$ at $t_{\mathrm{fan}}(\boldsymbol{\nabla})$ and $t_{\mathrm{ref}}(\boldsymbol{\vee})$, respectively. The shock intercepts the front, $x_{N}(-)$, at $t=t_{\text {col }}(\boldsymbol{\square})$. Uniform $\left(\mathrm{U}_{i}\right)$, Simple $\left(\mathrm{S}_{i}\right)$ and complex $\left(\mathrm{C}_{i}\right)$ regions are indexed by the order in which they appear. Online version in color. 

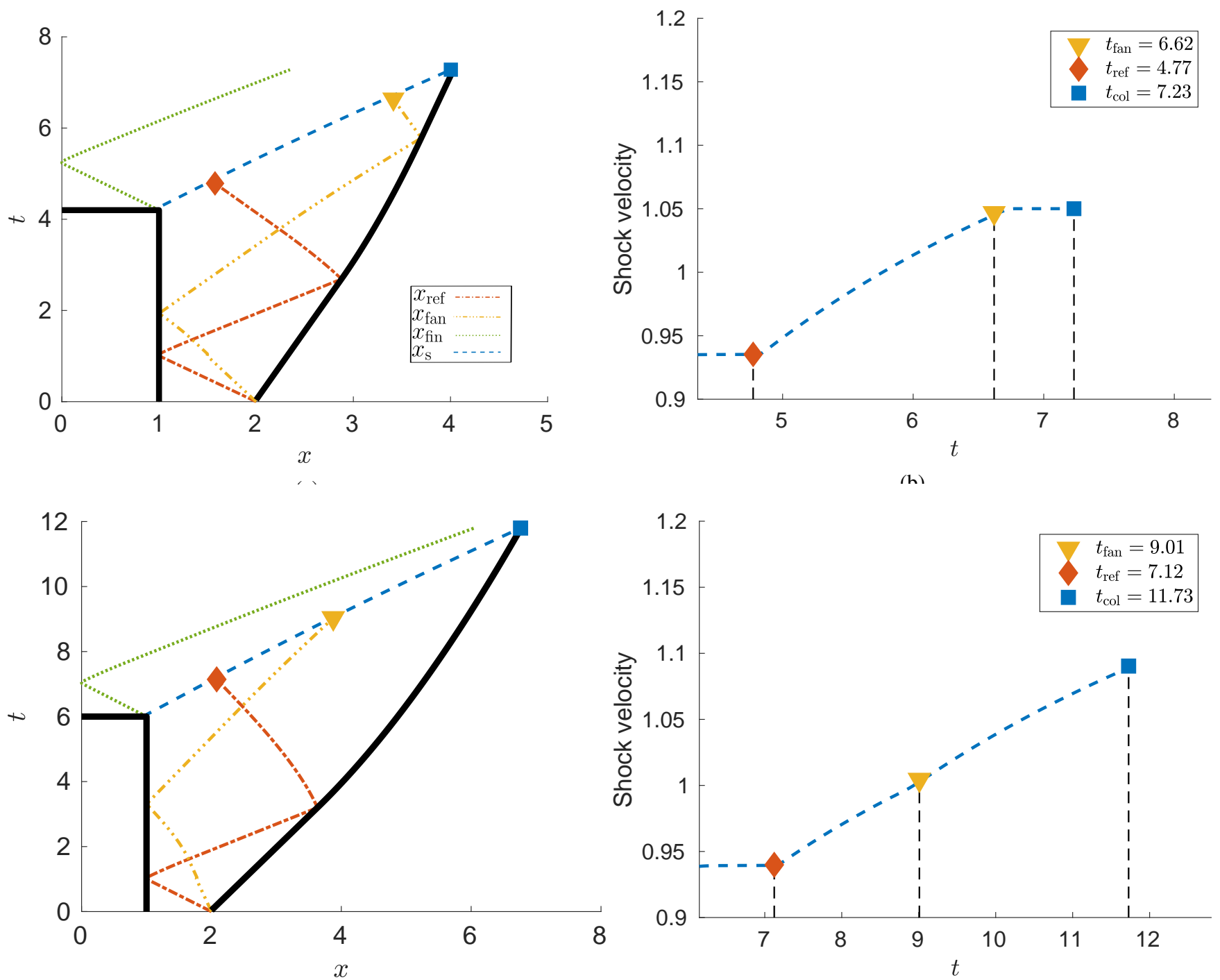

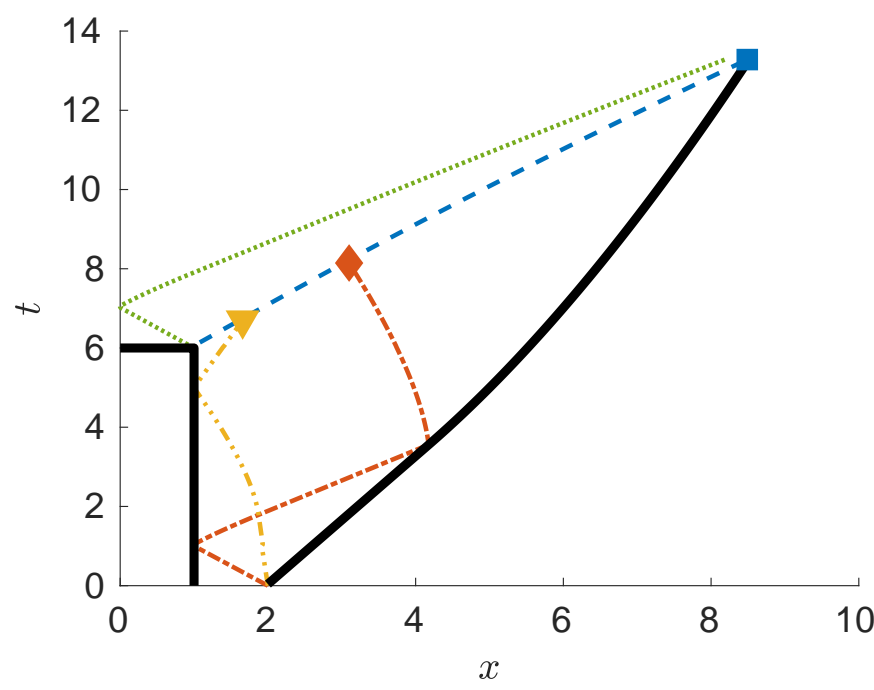

(e)

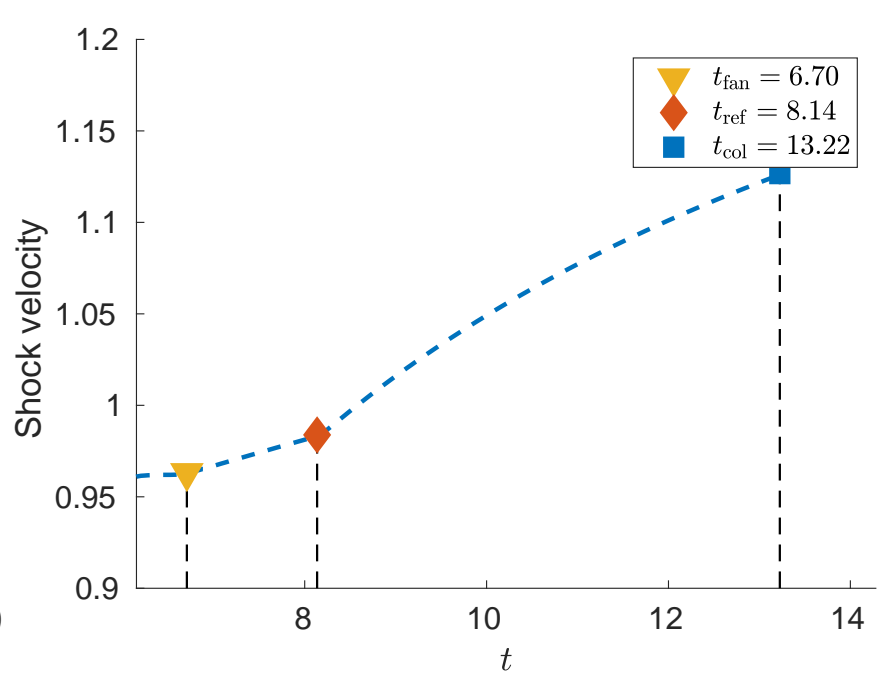

(f)

FIG. 7: Example $\mathrm{U}_{2} \mathrm{~N}$ cases for: $\mathrm{U}_{2} \mathrm{Ni}$, (a) \& (b) $\left(F r=0.4\right.$ and $\left.t_{\mathrm{re}}=4.2\right) ; \mathrm{U}_{2} \mathrm{Nii},(\mathrm{c}) \&(\mathrm{~d})\left(F r=0.6\right.$ and $\left.t_{\mathrm{re}}=5\right)$; and $\mathrm{U}_{2} \mathrm{Niii}$, (e) $\&$ (f) $\left(F r=0.9\right.$ and $\left.t_{\mathrm{re}}=5.8\right)$ : (a), (c), (e) Characteristic diagram displaying the flow boundaries $(-), x_{\mathrm{ref}}$ and $x_{\mathrm{fan}}(\cdots-)$, the shock $x_{s}(--)$ and $x_{\text {fin }}(\cdot)$; (b), (d), (f) Shock velocity for $t>t_{\text {re }} \cdot t_{\text {fan }}(\boldsymbol{\nabla}), t_{\text {ref }}(\bullet), t_{\text {col }}(\boldsymbol{\square})$ and $t_{\text {fin }}(\bullet)$ (a-f). The shock velocity is only linear in Uniform regions, §III A. Online version in color. 



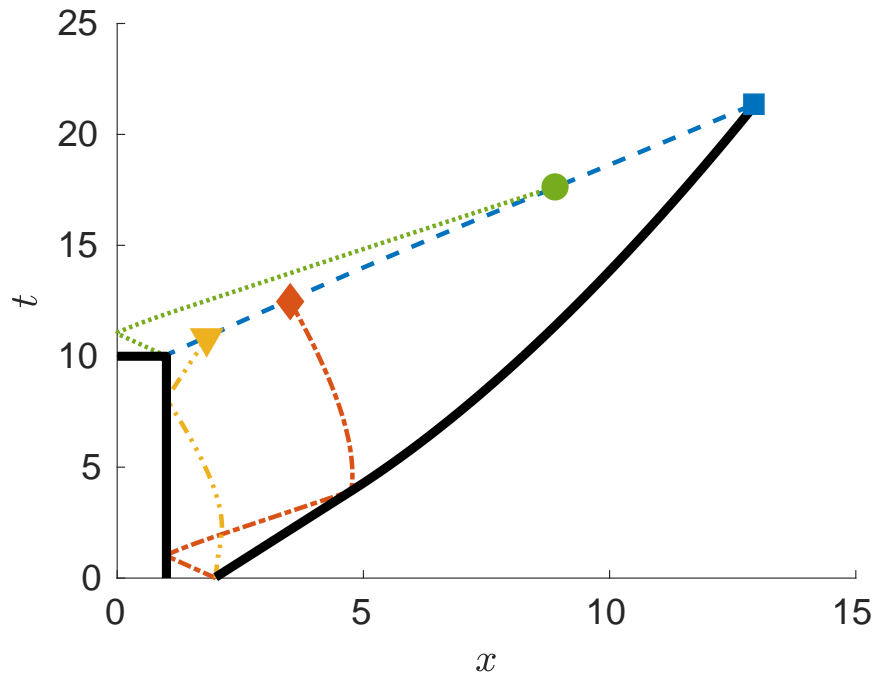

(c)

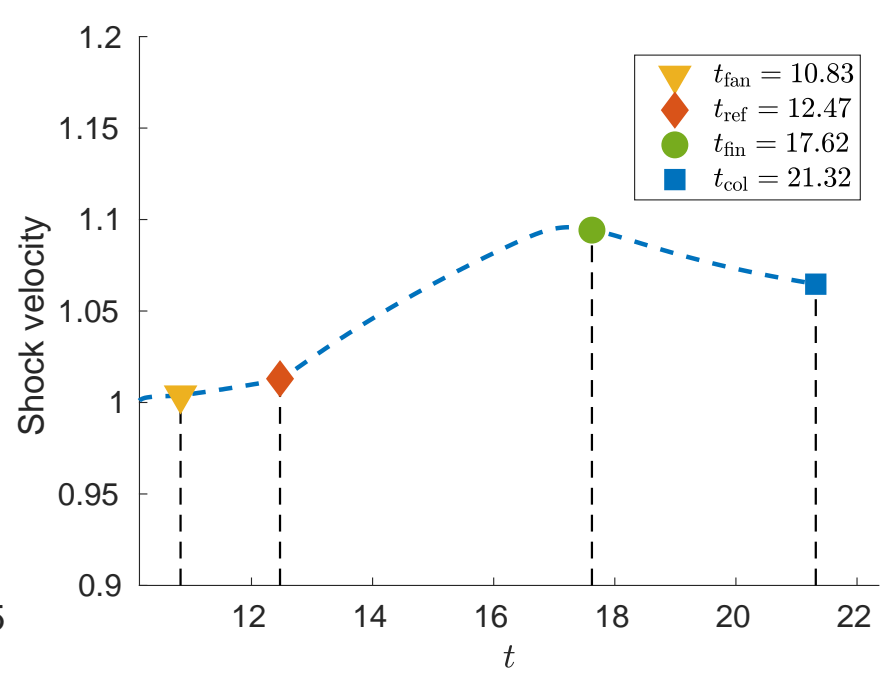

(d)

FIG. 8: Example $\mathrm{U}_{2}$ F cases for: $\mathrm{U}_{2}$ Fii, (c) \& (d) $\left(F r=0.9\right.$ and $\left.t_{\mathrm{re}}=10\right)$; and $\mathrm{U}_{2} F i i i$, (e) $\&$ (f) $\left(F r=1.1\right.$ and $\left.t_{\mathrm{re}}=10\right)$ : (a), (c) Characteristic diagram displaying the flow boundaries $(-), x_{\text {ref }}$ and $x_{\text {fan }}(\cdots-)$, the shock $x_{s}(--)$ and $x_{\text {fin }}(\cdot)$; (b), (d) Shock velocity for $t>t_{\mathrm{re}} . t_{\mathrm{fan}}(\boldsymbol{\nabla}), t_{\mathrm{ref}}(\bullet), t_{\mathrm{col}}(\boldsymbol{\square})$ and $t_{\mathrm{fin}}(\bullet)(\mathrm{a}-\mathrm{d})$. Online version in color.

run at these parameter values in order to resolve the parameter space accurately. The distinct regions of the parameter space are presented in Fig. 12, with the i, ii, and iii distinctions only shown for cases $\mathrm{C}_{1}, \mathrm{U}_{2}$ and $\mathrm{C}_{3}$. As $F r \rightarrow 0$, the boundaries collapse onto odd integers and the regions of complex shocks tend to zero. This is to be expected, as $x_{\text {ref }}$ and $x_{\text {fan }}$ coincide for $F r=0$, and, in fact, $u=0$ everywhere so positive and negative characteristics have gradient 1 or -1 , respectively. Cases $\mathrm{C}_{1}$ ii and $\mathrm{C}_{1}$ iii are the only two possible cases where the shock collides with $x_{\text {ref }}$ with $\alpha=2$. Our simulations reveal that neither of these cases exist.

The classification of each case determines exactly which three regions the shock travels through. the Rankine-Hugoniot conditions (21) \& (22) ensure that the shock velocity is constant in uniform regions where the shock is not affected by the finite length of the lock-box $\alpha^{-}=-2$. For shocks in simple wave regions, one of $\alpha^{+}$or $\beta^{+}$will take constant value given in equation (19) or (20), respectively, while the other will vary monotonically between two constant values dependent on $\mathrm{Fr}$ (from the neighboring uniform regions). In a complex region, the values of $\alpha^{+}$and $\beta^{+}$both vary between the constant values given in equations (19) \& (20).

Solving the Rankine-Hugoniot conditions (21) \& (22) numerically for a fixed $\alpha^{-}=2$ and varying $F r, \alpha^{+}$and $\beta^{+}$enables us to explore the regions of the flow where the shock $\dot{x}_{\mathrm{s}}$ may accelerating or decelerating. Studying the regions up to $S_{7}$ and $C_{6}$ revealed that the shock accelerates for all possible parameter values in regions of fixed $\alpha^{+}\left(\mathrm{S}_{3}, \mathrm{~S}_{5}\right.$ and $\left.\mathrm{S}_{7}\right)$ and for all values of $\alpha^{+}$above a critical value of the densimetric Froude number $F r_{c}$ in regions of fixed $\beta^{+}\left(\mathrm{S}_{2}, \mathrm{~S}_{4}\right.$ and $\mathrm{S}_{6}$ ). Similarly one variable was fixed and the other varied to determine $F r_{c}$-values for complex regions. This analysis re- 




(a)
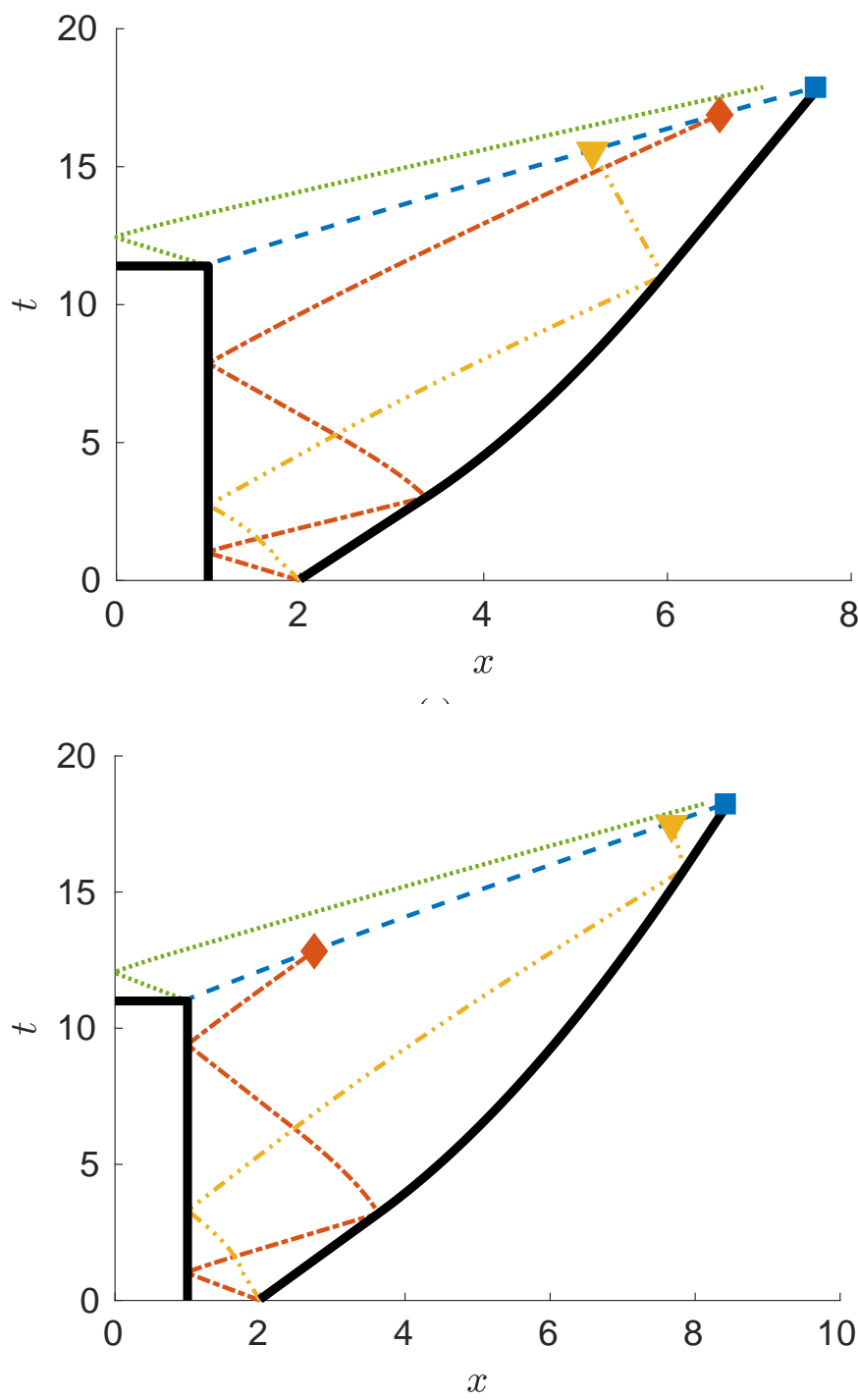

(e)


(d)

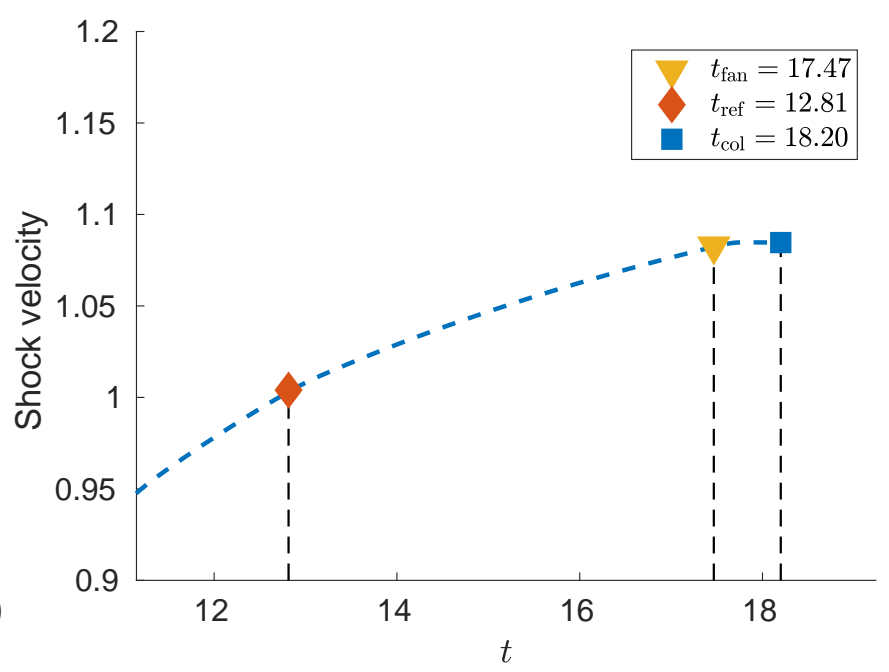

(f)

FIG. 9: Example $\mathrm{C}_{3} \mathrm{~N}$ cases for: $\mathrm{C}_{3} \mathrm{Ni}$, (a) \& (b) $\left(F r=0.4\right.$ and $\left.t_{\mathrm{re}}=10\right) ; \mathrm{C}_{3} \mathrm{Nii}$, (c) \& (d) $\left(F r=0.6\right.$ and $\left.t_{\mathrm{re}}=11.4\right)$; and $\mathrm{C}_{3} \mathrm{Niii}$, (e) \& (f) $\left(F r=0.7\right.$ and $\left.t_{\text {re }}=11\right)$ : (a), (c), (e) Characteristic diagram displaying the flow boundaries $(-), x_{\text {ref }}$ and $x_{\text {fan }}(\cdots-)$, the shock $x_{s}(--)$ and $x_{\text {fin }}(\cdot)$; (b), (d), (f) Shock velocity for $t>t_{\text {re }} \cdot t_{\text {fan }}(\boldsymbol{\nabla}), t_{\text {ref }}(\bullet), t_{\text {col }}(\boldsymbol{\square})$ and $t_{\text {fin }}(\bullet)$ (a-f). Online version in color. 

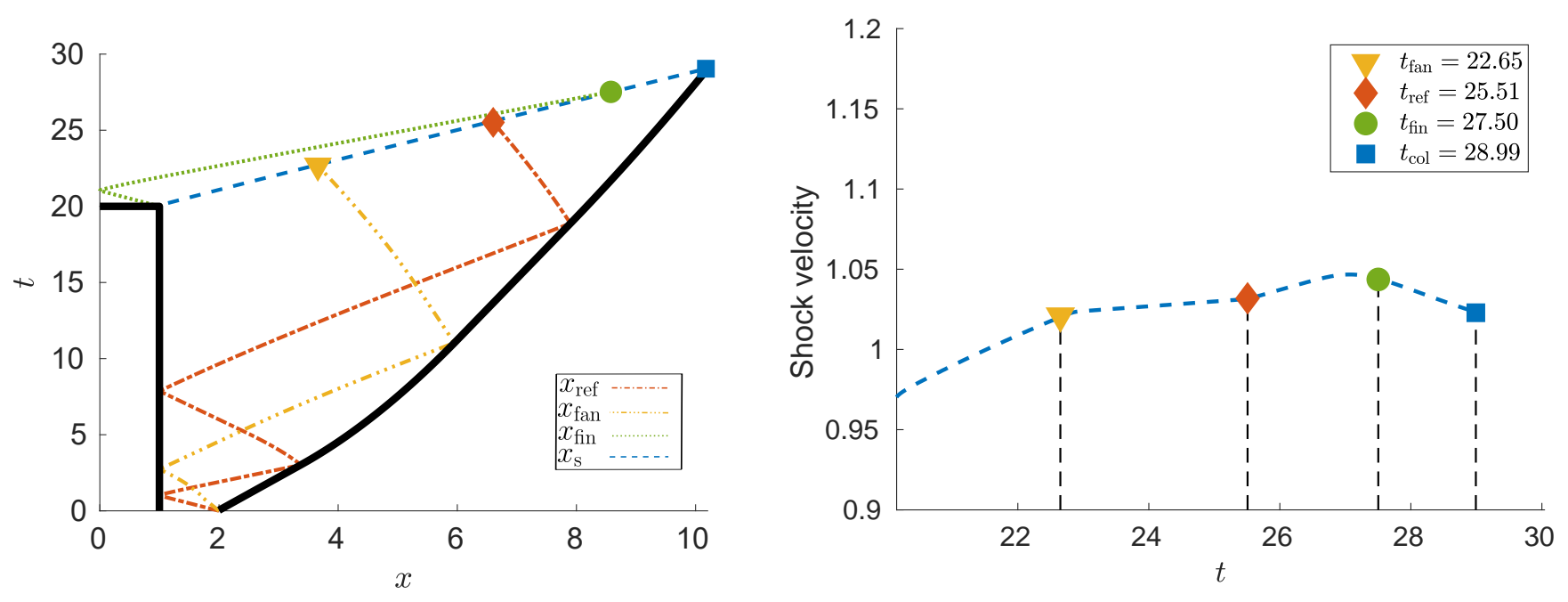

(b)
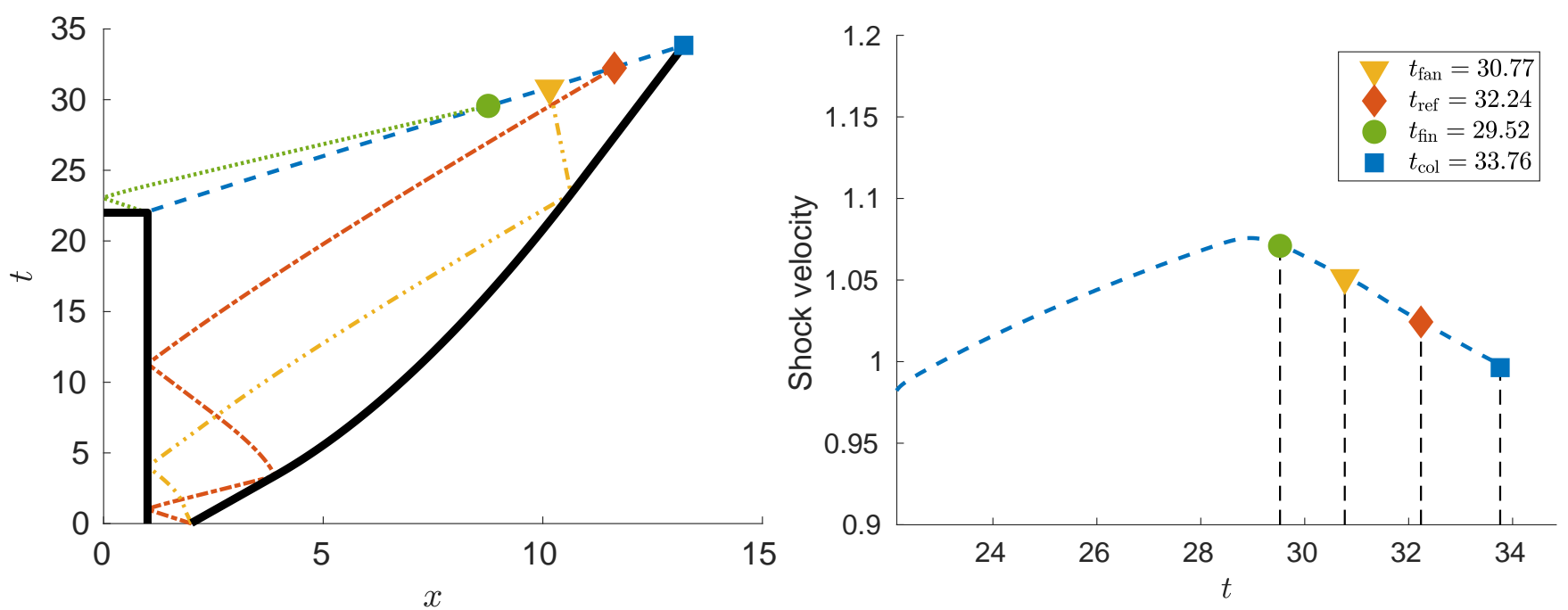

(d)

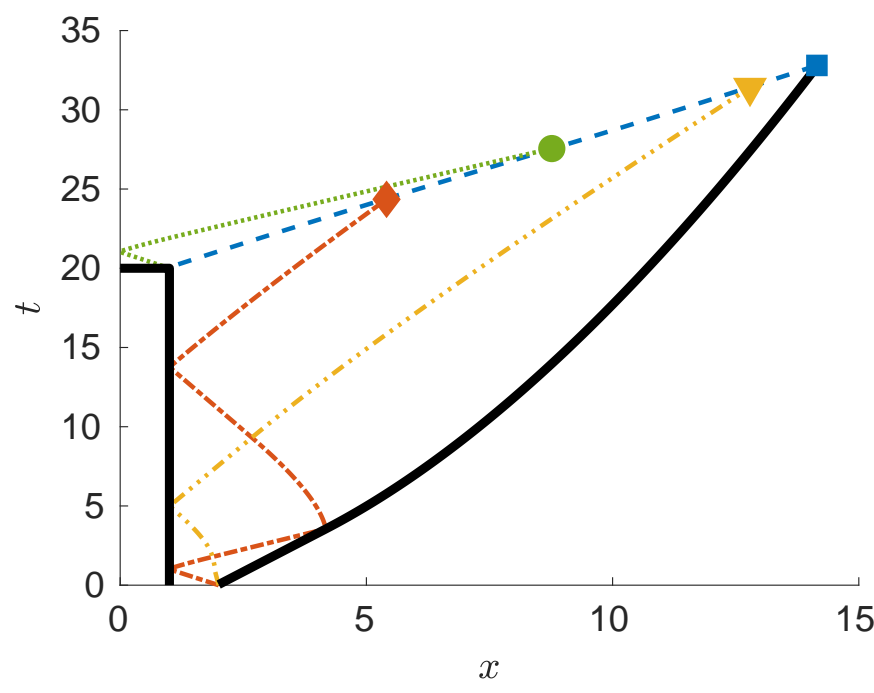

(e)



(f)

FIG. 10: Example $\mathrm{C}_{3} \mathrm{~F}$ cases for: $\mathrm{C}_{3} \mathrm{Fi}$, (a) \& (b) $\left(F r=0.3\right.$ and $\left.t_{\mathrm{re}}=20\right) ; \mathrm{C}_{3}$ Fii, (c) \& (d) $\left(F r=0.8\right.$ and $\left.t_{\mathrm{re}}=22\right)$; and $\mathrm{C}_{3} \mathrm{Fiii}$, (e) \& (f) $\left(F r=0.9\right.$ and $\left.t_{\mathrm{re}}=20\right)$ : (a), (c), (e) Characteristic diagram displaying the flow boundaries (-), $x_{\mathrm{ref}}$ and $x_{\mathrm{fan}}(\cdots-)$, the shock $x_{s}(--)$ and $x_{\mathrm{fin}}(\cdot)$; (b), (d), (f) Shock velocity for $t>t_{\mathrm{re}} \cdot t_{\mathrm{fan}}(\boldsymbol{\nabla}), t_{\mathrm{ref}}(\bullet), t_{\mathrm{col}}(\boldsymbol{\square})$ and $t_{\mathrm{fin}}(\bullet)$ (a-f). Online version in color. 
vealed that apart from case $\mathrm{C}_{3} \mathrm{Ni}$ in the $\mathrm{C}_{4}$ region and $\mathrm{C}_{3} \mathrm{Niii}$ in the $\mathrm{C}_{3}$ region, all the $\mathrm{Fr}_{c}$-values lie in the $\mathrm{F}$ region of the parameter space and therefore, all shocks in the $\mathrm{N}$ region up to case $\mathrm{U}_{6}$ have non-decreasing velocities, $\dot{x}_{\mathrm{s}}$. Sampling the $\mathrm{C}_{3}$ Niii case did not reveal any decelerating shocks. Decelerating shocks were found in $\mathrm{C}_{3} \mathrm{Ni}$ near the N-F boundary and just before the shock reaches the head. However, these were also observed for other cases near the N-F boundary. As the numerical method is not exact, the maximum shock velocity may arise just before the reflection of the backwards traveling disturbance $x_{\text {fin }}$ reaches the shock, Fig. 10b,d\&f. The velocity maximum occurs just before $t_{\text {fin }}$. This is believed to be a numerical artifact from the dissipative nature of the LaxWendroff scheme and that $\mathrm{N}$ shock do no necessarily decelerate.

The velocity of the flow behind the shock $u^{-}=\left(\alpha^{-}-\beta^{-}\right) / 2$ is related to the velocity of the shock through the Rankine-Hugoniot conditions (21) \& (22). The simulations reveal that the acceleration of the flow behind the shock has the same sign as the shock acceleration, except when the shock is propagating through $S_{2 n}$ regions of the flow, i.e. regions where $\beta^{+}$takes a constant value. In these regions the acceleration has the opposite polarity. Critically, this means that an increasing shock speed is equivalent to an increasing fluid velocity behind the shock for all other regions in the single release flow.

Depth $h$ and velocity $u=m / h$ are compared for cases $\mathrm{U}_{2} \mathrm{Ni}$ and $\mathrm{C}_{3} \mathrm{Ni}$ in Fig. 11. The position of the curves $x_{\text {ref }}(\checkmark)$ and $x_{\text {fan }}(\boldsymbol{\nabla})$ are displayed along the $x$-axis to highlight the boundaries between uniform, simple, and complex regions. After the second release the position of the shock $(\boldsymbol{\square})$ and $x_{\text {ref }}(\bullet)$ are also shown. Both cases are for the same $F r$, so the initial dynamics are identical. After the second release, the flow depth is deeper, but slower, behind the shock for the $\mathrm{U}_{2} \mathrm{Na}$ case when compared with the $\mathrm{C}_{3} \mathrm{Ni}$ case. When the shock reaches the head of the flow the expected constant depth and velocity is observed for the $\mathrm{U}_{2} \mathrm{Na}$ case. Whereas for the $\mathrm{C}_{3} \mathrm{Ni}$ case, the velocity increases to a maximum at the head of the flow. This maximum is larger than the maximum velocity for the $\mathrm{U}_{2} \mathrm{Na}$ case.

\section{Momentum of the Head}

Both the destructive potential, i.e. the amount of damage it can cause, of the gravity current and its run-out length can be affected by the fluid momentum at the head of the current $m\left(x_{N}, t\right)$. In Fig. 13 we compare the momentum at the time when the shock collides with the head at the same time, $m\left(x_{N}, t_{\text {col }}\right)$, for four different Froude numbers $F r$ against pulse separation times $t_{\mathrm{re}}$. Also shown on this plot is the corresponding momentum for a single release of twice the size at $(x, t)=\left(x_{N}, t_{\mathrm{col}}\right)$. For a fixed $F r$, the momentum is significantly lower for a single release than the corresponding double release. Although both cases contain the same amount of material, the depth at the head of the flow starts decreasing later for the single release and therefore the dissipation is larger. Further, the dissipation is significantly larger at the head of the flow than at the shock.

In contrast to the single release, where increasing the densimetric Froude number decreases the dissipation at the head, plotted against separation time the momentum in the corresponding double release is lower for higher densimetric Froude numbers. This is a consequence of the head moving faster for higher Froude numbers. If instead the momentum at $t=t_{\mathrm{col}}$ is plotted against the position of the head $x_{N}\left(t_{\mathrm{col}}\right)$, Fig. $13 \mathrm{~b}$ the expected trend is observed with higher Froude numbers being less dissipative. Critically, although the distinction is less with larger Froude numbers $F r$, a single release event is more dissipative than a double release.

\section{IMPLICATIONS}

We can now reflect on the implications of our model findings on the geophysical events discussed in the introduction.For compositional flows, the particle size distribution is the dominant control on when erosion and deposition rates are in balance ${ }^{6}$. For dilute flows, this equilibrium point defines a particle concentration above or below which the flow is erosional or depositional, respectively. The models discussed in Dorrell et al. ${ }^{6}$ are increasing functions of the shear velocity at the bed $u_{*}$, which can be empirically modeled as proportion to, or related to a positive power of the depth-averaged velocity $u=m / h^{37-39}$. Thus, in regions where the flow speed is increasing it is more net erosional and conversely for regions where the flow speed is decreasing it is more net depositional. When the particle concentration is large, for example lahars and landslides, the particles are in constant contact and suspended by matrix strength rather than the fluid. Therefore there is no simple relationship between erosion and deposition rates and the acceleration of the flow for these flows. Instead erosion and deposition are controlled by a stress balance at the bed $^{40}$

The hazard assessments conducted for the Soufriére Hills Volcano, Montserrat assume a continuous steady release of material from the lava dome during the 1997 eruption creating a pyroclastic surge flow ${ }^{7}$. However, at the 1997 eruption the dome failed retrogressively producing a continuous release with three distinct peaks in volume flux identified as distinct pulses. Sedimentation from pyroclastic surges can create a complex flow structure with a dense, basal pyroclastic flow component with high-particle concentration, which is overlain by and an upper, less dense pyroclastic surge. In the 1997 event, $1.4 \mathrm{~km}$ downstream from source the third pyroclastic flow associated with the third pulse overspilled the drainage channel and went on to hit the villages of Streatham and Windy Hill. Despite the bend coinciding with a constriction in the channel cross-sectional area these villages were not considered at risk. However, the previous two pulses had left significant deposits thus increasing the risk of surge detachment and overspilling.

The pulsed nature of this flow affected its run-out and the inundation zone of this flow when compared to the a continuous release of the same size ${ }^{7}$. The shallow-water model discussed in this paper suggests that the separation time between 

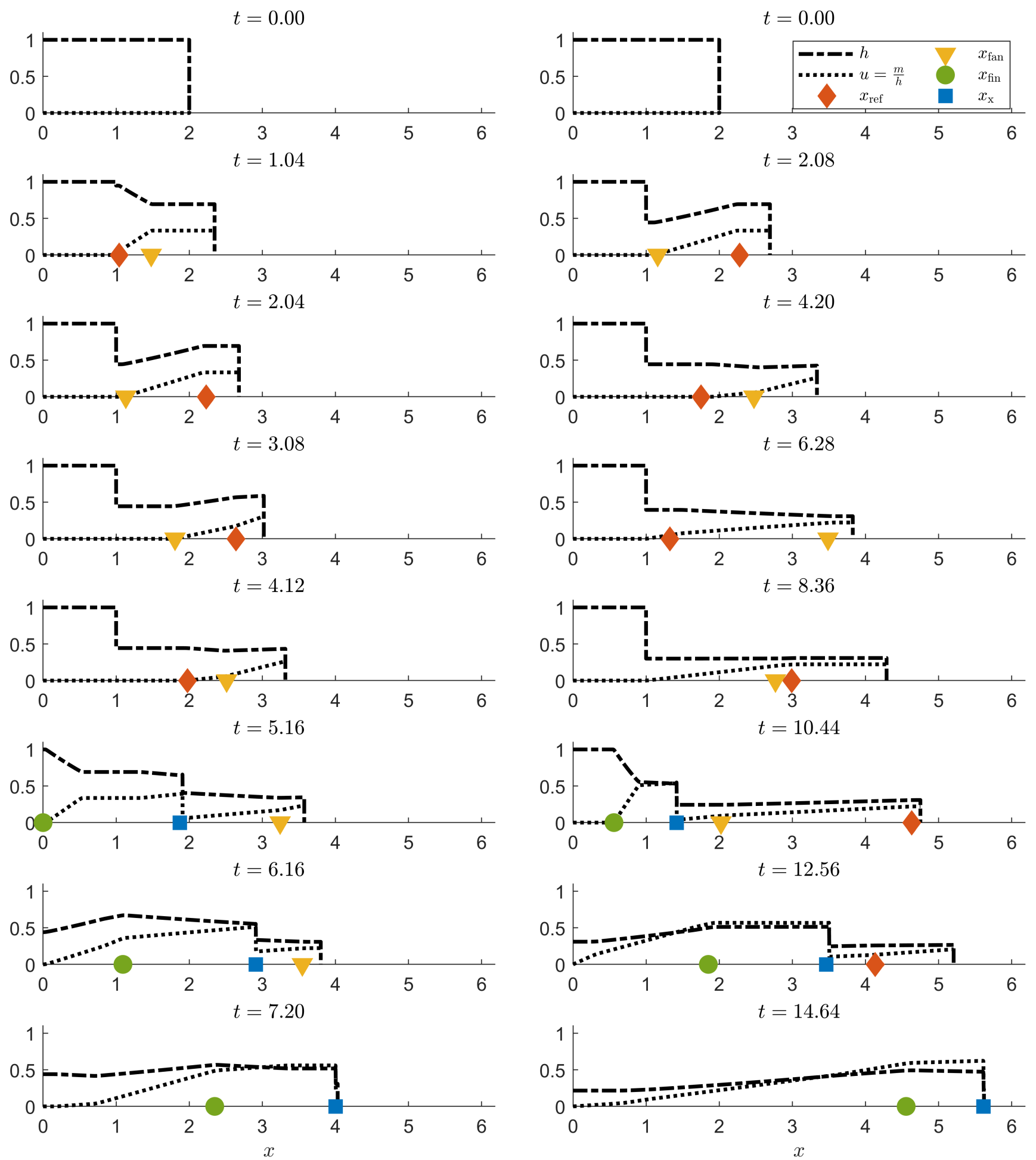

FIG. 11: Depth $h(--\cdot)$ and velocity $u=m / h(\cdots)$ profiles at eight equally-spaced time steps until the shock reaches the head of the current for the following cases: $\mathrm{U}_{2} \mathrm{Ni}$ (left) and $\mathrm{C}_{2} \mathrm{Ni}$ (right). Positions of the curves $x_{\text {ref }}(\checkmark)$ and $x_{\text {ref }}(\boldsymbol{\nabla})$ are displayed on the $x$-axis until they intersect the shock $x_{\mathrm{s}}(\boldsymbol{\square})$. The position of $x_{\mathrm{fin}}(\bullet)$ is also displayed on the $x$-axis. 


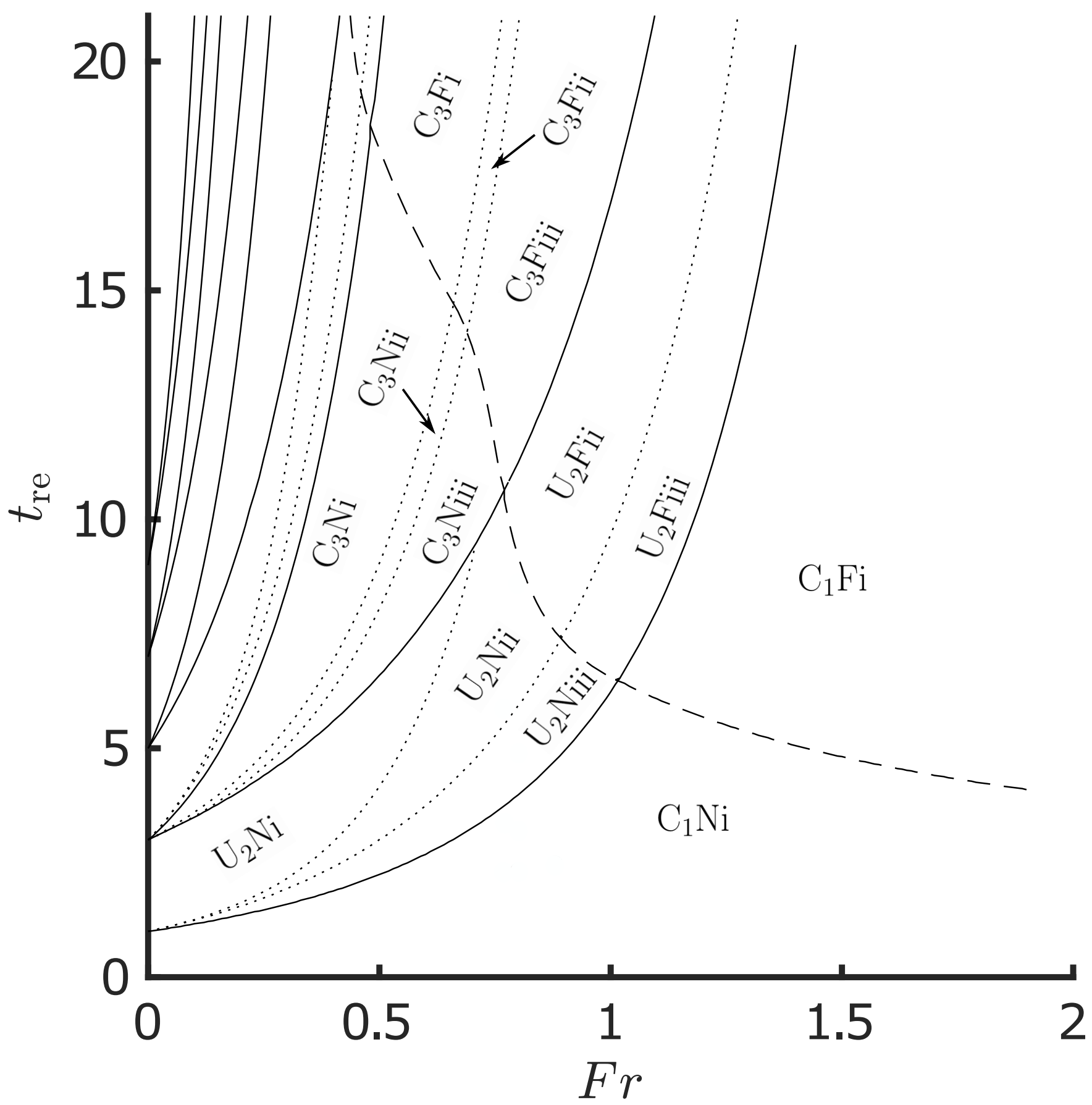

FIG. 12: Structure of the $\left(F r, t_{\mathrm{re}}\right)$ parameter space up to $\mathrm{C}_{3}$ shocks. Numerically determined boundaries between regions (-), sub-regions $(\cdots)$ and N/F (- $)$ are shown.

the pulses may have a crucial affect on whether the pulses were erosional or depositional at a particular location. Pulses that are close together (case $\mathrm{N}$ ) are non-decelerating and may entrain more material downstream increasing the depth of the channel or increase the velocity/momentum of the flow. For large separation times the pulses may transition from erosional to depositional downstream filling in the channel and making it more susceptible to future flows overspilling.

\section{CONCLUSIONS}

We have explored the affect of pulses on gravity current propagation using an extension of the shallow-water model for the single lock-release case studied by $\mathrm{Hogg}^{27}$. The range of solutions are classified in terms of two parameters: the Froude number at the head of the current, Fr, and a dimensionless pulse separation time, $t_{\mathrm{re}}$. For $t_{\mathrm{re}} \leq 1$ the problem is identical to a single release, whilst the limits $t_{\mathrm{re}} \rightarrow \infty$ and $t_{\mathrm{re}} \rightarrow 1^{+}$ 



(b)

FIG. 13: Computed momentum at the head of the flow when the shock arrives, $m\left(x_{N}, t_{\mathrm{col}}\right)$, for four Froude numbers $F r=(0.5,0.75,1,1.25)$ in increments of 2 pulse separation times $t_{\mathrm{re}}(-)$ against: (a) pulse separation time $t_{\mathrm{re}}$; (b) displacement of the head at the time of collision, $t_{\mathrm{col}}$. Momentum at the collision time, $t_{\mathrm{col}}$, for a single release of twice the size $t_{\mathrm{re}}<1(\cdots)$ is also shown. Online version in color.

correspond to two independent events and a single event of twice the volume, respectively. For intermediate values of $t_{\text {re }}$ the order the pulse intersects three curves $x_{\text {ref }}, x_{\mathrm{fan}}$ and $x_{\mathrm{fin}}$, qualitatively determine its propagation velocity. Critically, the pulse has non-negative acceleration before it intersects $x_{\text {fin }}$ and negative acceleration thereafter. For small values of $t_{\mathrm{re}}$ and/or small values of the Froude number, $F r, x_{\text {fin }}$ does not intersect the pulse before it reaches the front, Fig. 12.

Variations in pulse velocity affect the rate of energy dissipa- tion, and thus of the energy transferred through to the head of the current, which may enable the flow to transition from laminar to turbulent behavior. For pulse-prone, Boussines compositional flows such as pyroclastic flows, the dynamics of the flow depend on dynamics of the release and the changes in flow velocity may have implications for hazard prediction models, which sometimes neglect the release dynamics and the subsequent pulses created.

\section{ACKNOWLEDGMENTS}

PAA would like to Acknowledge EPSRC for providing funding for this work through the Centre for Doctoral Training in Fluid Dynamics grant number EP/L01615X/1 and through the Turbidite Research Group: AkerBP; BP; ConocoPhilips; Equinor; ENI; Hess; Murphy Oil Corporation; OMV and Shell, and to Marius Ungarish for advice on the theoretical modeling. This work was undertaken on ARC3, part of the High Performance Computing facilities at the University of Leeds, UK.

\section{Appendix}

Verification of the above scheme was conducted by comparing simulations of a single lock-release problem to the solution presented in $\operatorname{Hogg}^{27}$. Depth and velocity profiles at $t=1$ are compared for fixed $\Delta t$ and varying $\Delta x$ and vice versa, Fig. 14. The error of the variable $E_{f}$ is calculated via the $\ell_{1}$-norm of the variable

$$
E_{f}=\frac{\ell_{1}\left(f_{a}-f_{n}\right)}{N_{\text {int }}}=\frac{1}{N_{\text {int }}} \sum_{k=1}^{N_{\text {int }}}\left|f_{a}-f_{n}\right|,
$$

where $f=h$ or $f=u \equiv m / h$ and $N_{\text {int }}$ is the number of nodes over the averaging interval. The numerical, subscript $n$, and analytical, subscript $a$, solutions are interpolated onto an equally spaced grid with $N_{\text {int }}=10^{4}$. For fixed $\Delta x$ the error quickly converges to the spatial error and therefore a numerical solution $\Delta t=10^{-6}$ and $\Delta x=0.005$ is used instead of the analytical solution for comparison. After $t=1$, complex regions start appearing in the characteristic space and a depth and velocity profile across the length of the current cannot be explicitly written down everywhere. However, the curve $x_{\text {ref }}$ has been expressed in closed form in equation (18) and this expression is compared to a positive characteristic emanating from $(1,1)$ calculated from the numerical solution. An excellent agreement between the analytical and numerical expression for $x_{\text {ref }}$ is observed, Fig. 15. Although the Lax-Wendroff finite-difference scheme is formally second order accurate, the model verification suggests that it is first order accurate in both time and space. This is to be expected as the analytic solution of the dam break does not have a continuous derivative everywhere. Although not displayed here, for lower resolutions the contribution to the error is largest at $x=0$ and $x=1$. 

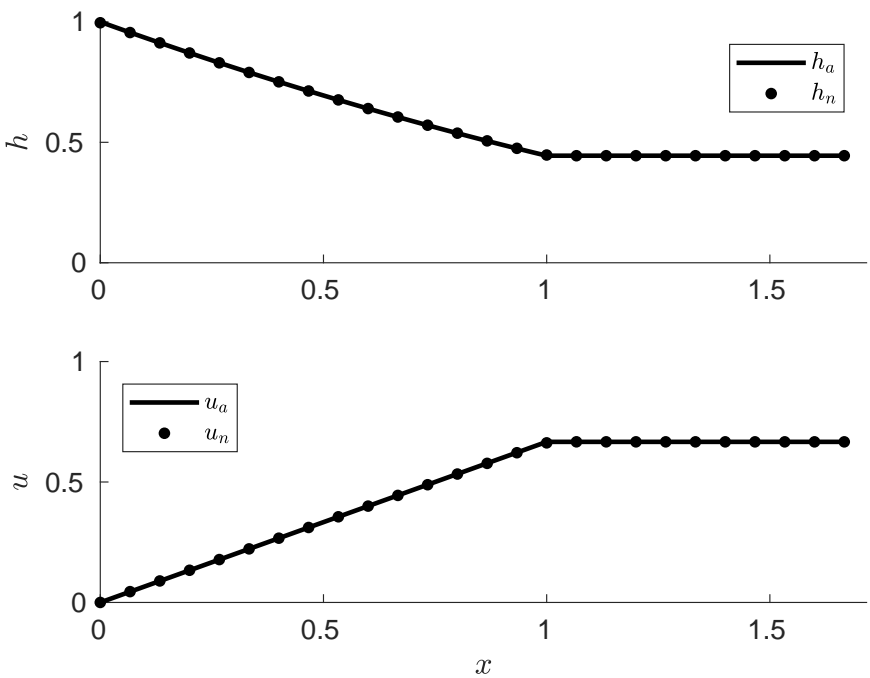

(a)


(b)

FIG. 14: (a) Depth and velocity profiles at $t=1$ for a single release problem $\left(\Delta x=0.0002, \Delta t=10^{-5}, F r=1\right)$. (b) Error in depth and velocity for fixed $\Delta t=10^{-5}$ (left) and fixed $\Delta x=0.005$ (right). Straight lines drawn between the end points have $E_{h} \sim \Delta t^{0.85}, E_{u} \sim \Delta t^{0.79}, E_{h} \sim \Delta x^{1.04}$ and

$$
E_{u} \sim \Delta x^{1.04} \text {. }
$$

${ }^{1}$ J. E. Simpson, Gravity Currents: In the Environment and the Laboratory (Cambridge University Press, 1997).

${ }^{2}$ T. Mulder and J. Alexander, "The physical character of subaqueous sedimentary density flows and their deposits," Sedimentology 48, 269-299 (2001).

${ }^{3}$ T. Nakajima and Y. Kanai, "Sedimentary features of seismoturbidites triggered by the 1983 and older historical earthquakes in the eastern margin of the Japan Sea," Sedimentary Geology 135, 1-19 (2000).

${ }^{4}$ P. D. W. Haughton, "Deposits of deflected and ponded turbidity currents, Sorbas Basin, southeast Spain,” Journal of Sedimentary Research 64, 233 246 (1994).

${ }^{5}$ B. Wang, P. J. Vardon, and M. A. Hicks, "Investigation of retrogressive and progressive slope failure mechanisms using the material point method," Computers and Geotechnics 78, 88-98 (2016).

${ }^{6}$ R. M. Dorrell, L. A. Amy, J. Peakall, and W. D. McCaffrey, "Particle size distribution controls the threshold between net sediment erosion and depo-

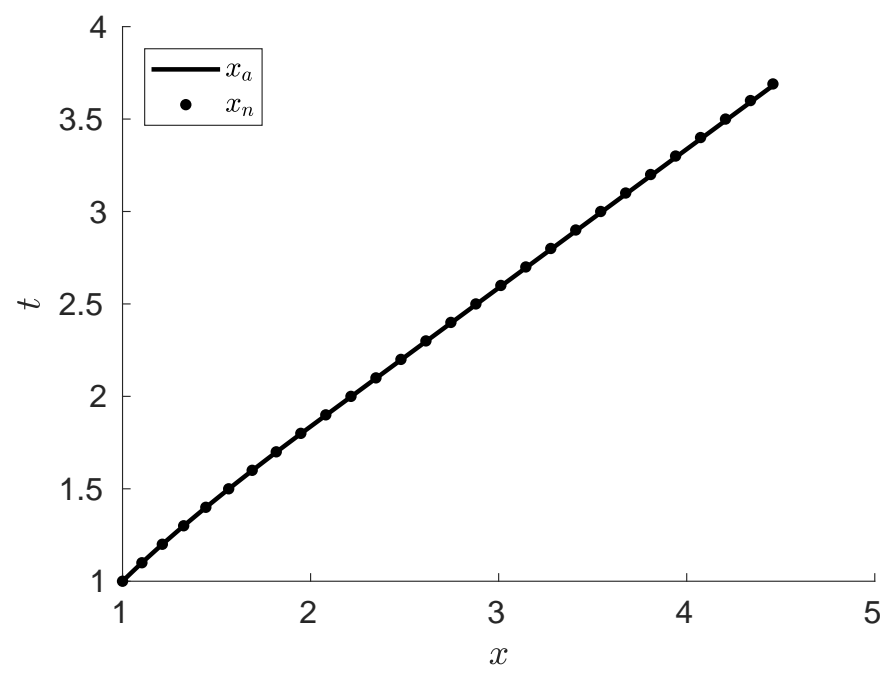

FIG. 15: Comparison between the analytical expression and the numerically-computed $x_{\text {ref }}$ between $(1,1)$ and the point that it intersects the head.

sition in suspended load dominated flows," Geophysical Research Letter 45, 1443-1452 (2018)

${ }^{7}$ S. C. Loughlin, E. S. Calder, A. Clarke, P. D. Cole, R. Luckett, M. T. Mangan, D. M. Pyle, R. S. J. Sparks, B. Voight, and R. B. Watts, "Pyroclastic flows and surges generated by the 25 June 1997 dome collapse, Soufrière Hills Volcano, Montserrat," Geological Society, London, Memoirs 21, 191209 (2002)

${ }^{8}$ D. J. W. Piper, P. Cochonat, and M. L. Morrison, "The sequence of events around the epicentre of the 1929 Grand Banks earthquake: initiation of debris flows and turbidity current inferred from sidescan sonar,' Sedimentology 46, 79-97 (1999).

${ }^{9}$ E. Gonzalez-Juez, E. Meiburg, and G. Constantinescu, "Gravity currents impinging on bottom-mounted square cylinders: flow fields and associated forces," Journal of Fluid Mechanics 631, 65-102 (2009).

${ }^{10}$ A. T. Dengler, P. Wilde, E. K. Noda, and W. R. Normark, "Turbidity currents generated by hurricane Iwa," Geo-Marine Letters 4, 5-11 (1984).

${ }^{11}$ B. C. Heezen and M. Ewing, "Turbidity currents and submarine slumps, and the 1929 Grand Banks earthquake,' American Journal of Science 250, 849-873 (1952).

${ }^{12} \mathrm{P}$. Weimer, R. M. Slatt, and R. Bouroullec, Introduction to the Petroleum Geology of Deep-water Settings, Vol. 57 (AAPG, 2007).

${ }^{13}$ C. Goldfinger, S. Galer, J. Beeson, T. Hamilton, B. Black, C. Romsos, J. Patton, C. H. Nelson, R. Hausmann, and A. Morey, "The importance of site selection, sediment supply, and hydrodynamics: A case study of submarine paleoseismology on the Northern Cascadia margin, Washington USA," Marine Geology 384, 4-46 (2017).

${ }^{14}$ V. L. Ho, R. M. Dorrell, G. M. Keevil, A. D. Burns, and W. D. McCaffrey, "Scaling analysis of multipulsed turbidity current evolution with application to turbidite interpretation," Journal of Geophysical Research: Oceans 123, 3668-3684 (2018).

${ }^{15}$ D. P. Hoult, "Oil spreading on the sea," Annual Review of Fluid Mechanics 4, 341-368 (1972).

${ }^{16}$ H. E. Huppert and J. E. Simpson, “The slumping of gravity currents," Journal of Fluid Mechanics 99, 785-799 (1980).

${ }^{17} \mathrm{H}$. E. Huppert, "The propagation of two-dimensional and axisymmetric viscous gravity currents over a rigid horizontal surface," Journal of Fluid Mechanics 121, 43-58 (1982).

${ }^{18}$ J. O. Shin, S. B. Dalziel, and P. F. Linden, "Gravity currents produced by lock exchange," Journal of Fluid Mechanics 521, 1-34 (2004).

${ }^{19}$ R. J. Lowe, J. W. Rottman, and P. F. Linden, "The non-Boussinesq lockexchange problem. Part 1. Theory and experiments," Journal of Fluid Mechanics 537, 101-124 (2005). 
${ }^{20}$ V. L. Ho, R. M. Dorrell, G. M. Keevil, A. D. Burns, and W. D. McCaffrey, "Pulse propagation in turbidity currents," Sedimentology 65, 620-637 (2018).

${ }^{21}$ V. L. Ho, R. M. Dorrell, G. M. Keevil, R. E. Thomas, A. D. Burns, J. H. Baas, and W. D. McCaffrey, "Dynamics and deposition of sedimentbearing multi-pulsed flows and geological implication," (2019).

${ }^{22}$ J. A. Fay, "Physical processes in the spread of oil on a water surface," International Oil Spill Conference Proceedings 1971, 463-467 (1971) https://doi.org/10.7901/2169-3358-1971-1-463.

${ }^{23}$ A. J. Hogg, M. A. Hallworth, and H. E. Huppert, "On gravity currents driven by constant fluxes of saline and particle-laden fluid in the presence of a uniform flow," Journal of Fluid Mechanics 539, 349-385 (2005).

${ }^{24}$ G. B. Whitham, Linear and nonlinear waves (John Wiley \& Sons, 2011).

${ }^{25}$ C. G. Johnson, A. J. Hogg, H. E. Huppert, R. S. J. Sparks, J. C. Phillips, A. C. Slim, and M. J. Woodhouse, "Modelling intrusions through quiescent and moving ambients," Journal of Fluid Mechanics 771, 370-406 (2015).

${ }^{26}$ M. B. Abbott, "On the spreading of one fluid over another: part 2," La Houille Blanche 6, 827-836 (1961)

${ }^{27}$ A. J. Hogg, "Lock-release gravity currents and dam-break flows," Journal of Fluid Mechanics 569, 61-87 (2006).

${ }^{28}$ T. Von Karman, "The engineer grapples with nonlinear problems," Bulletin of the American Mathematical Society 46, 615-683 (1940).

${ }^{29}$ T. B. Benjamin, "Gravity currents and related phenomena," Journal of Fluid Mechanics 31, 209-248 (1968).

${ }^{30} \mathrm{Z}$. Borden and E. Meiburg, "Circulation based models for Boussinesq gravity currents," Physics of Fluids 25, 101301 (2013).
${ }^{31}$ M. Ungarish and A. J. Hogg, "Models of internal jumps and the fronts of gravity currents: unifying two-layer theories and deriving new results," Journal of Fluid Mechanics 846, 654-685 (2018).

${ }^{32} \mathrm{M}$. Ungarish, "Intrusive gravity currents in a stratified ambient: shallowwater theory and numerical results," Journal of Fluid Mechanics 535, 287 323 (2005).

${ }^{33} \mathrm{M}$. Ungarish, "A general solution of Benjamin-type gravity current in a channel of non-rectangular cross-section," Environmental Fluid Mechanics 12, 251-263 (2012).

${ }^{34}$ R. T. Bonnecaze, H. E. Huppert, and J. R. Lister, "Particle-driven gravity currents," Journal of Fluid Mechanics 250, 339-369 (1993).

${ }^{35}$ J. J. Stoker, Water waves: The Mathematical Theory with Applications (John Wiley \& Sons, 2011).

${ }^{36}$ T. H. Ellison and J. S. Turner, "Turbulent entrainment in stratified flows," Journal of Fluid Mechanics 6, 423-448 (1959).

${ }^{37}$ S. Soares-Frazão and Y. Zech, "Hllc scheme with novel wave-speed estimators appropriate for two-dimensional shallow-water flow on erodible bed,' International journal for numerical methods in fluids 66, 1019-1036 (2011)

${ }^{38}$ G. Simpson and S. Castelltort, "Coupled model of surface water flow, sediment transport and morphological evolution," Computers \& Geosciences 32, 1600-1614 (2006)

${ }^{39}$ S. Yang, Y. An, and Q. Liu, "A two-dimensional layer-averaged numerical model for turbidity currents," Geological Society, London, Special Publications 477, 439-454 (2019).

${ }^{40} \mathrm{R}$. M. Iverson and C. Ouyang, "Entrainment of bed material by Earthsurface mass flows: Review and reformulation of depth-integrated theory," Reviews of Geophysics 53, 27-58 (2015). 


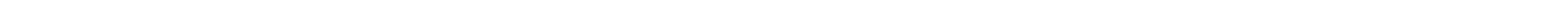









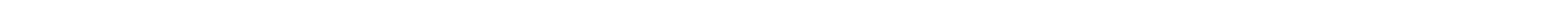




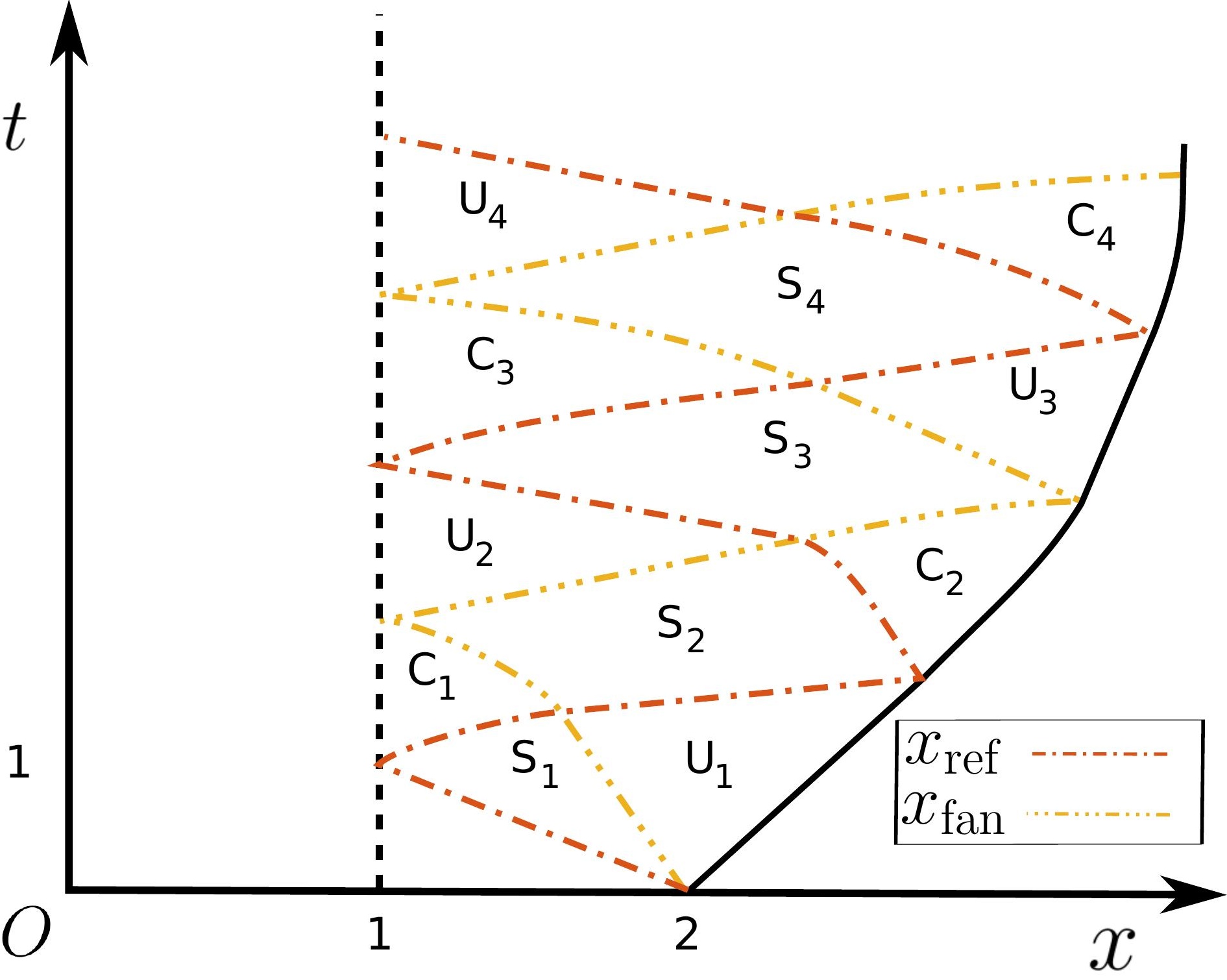




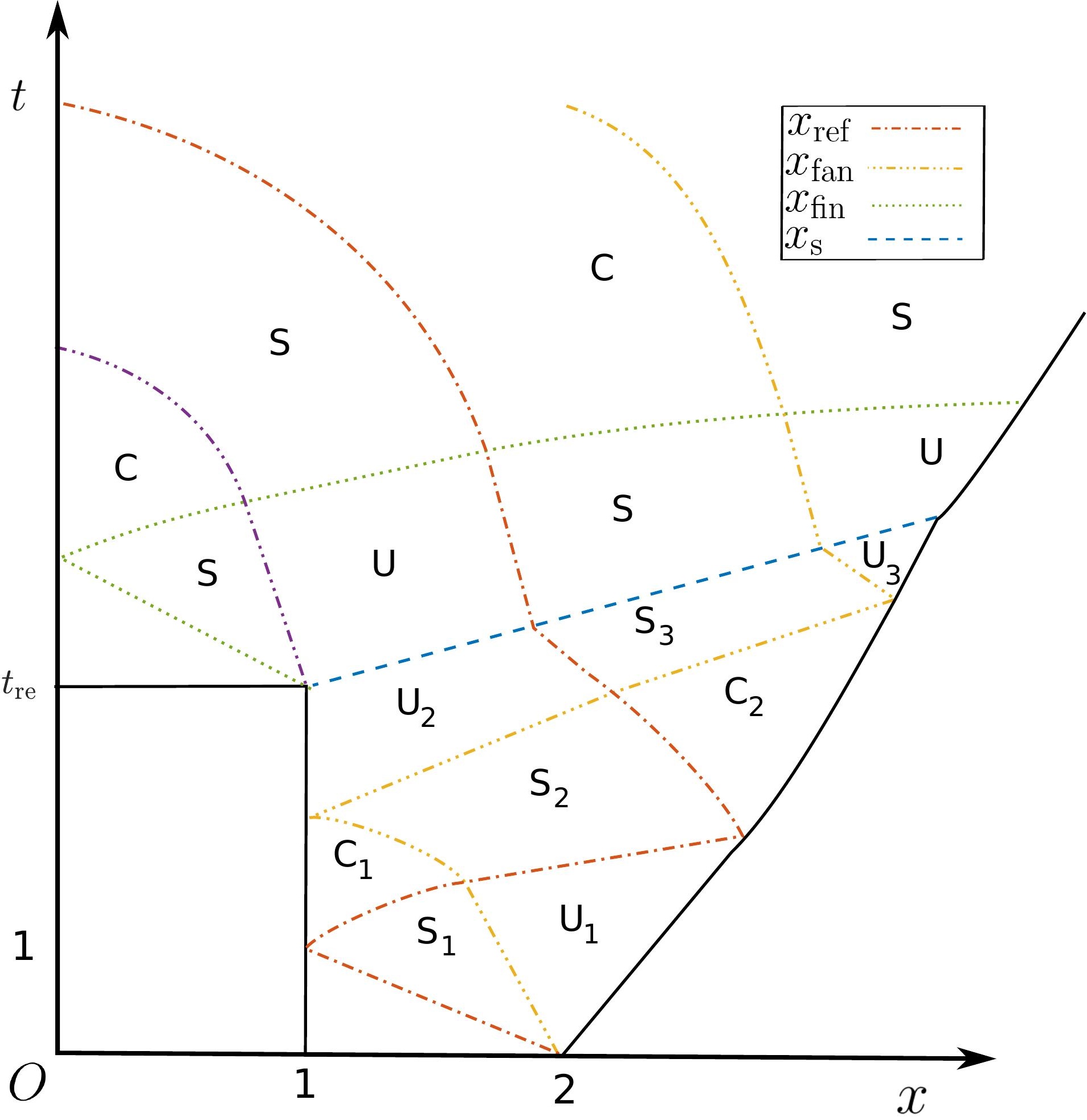









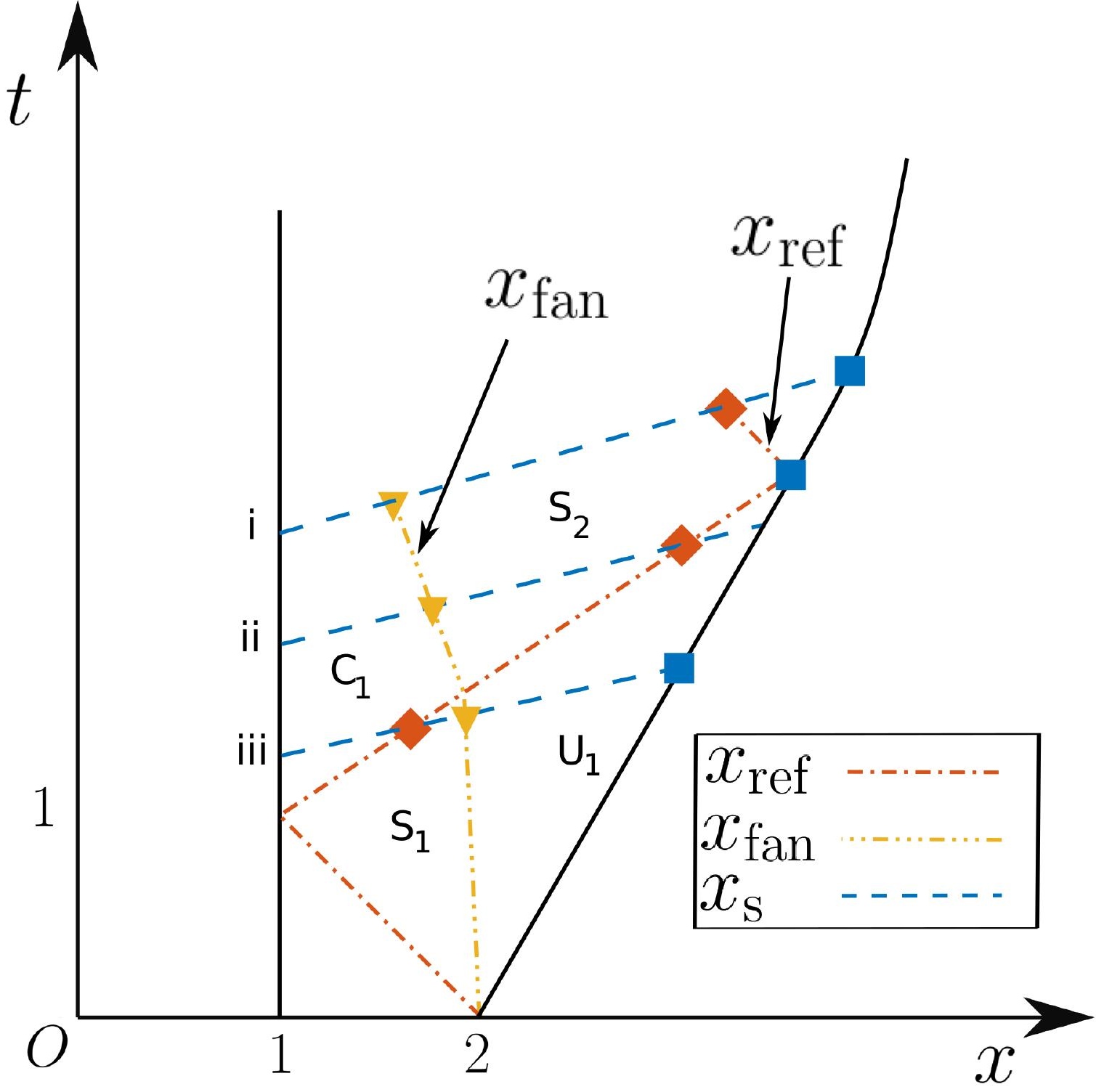




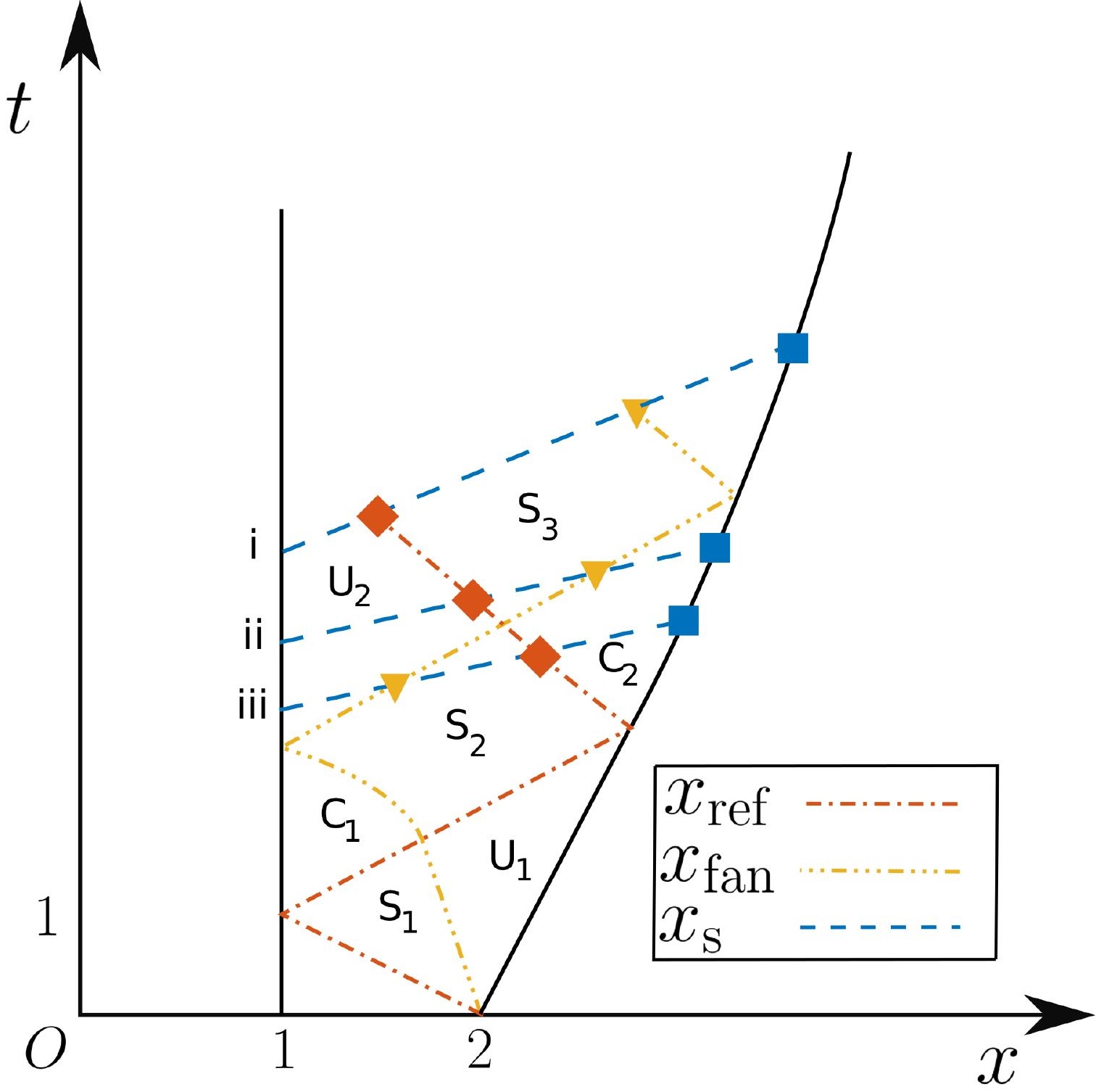




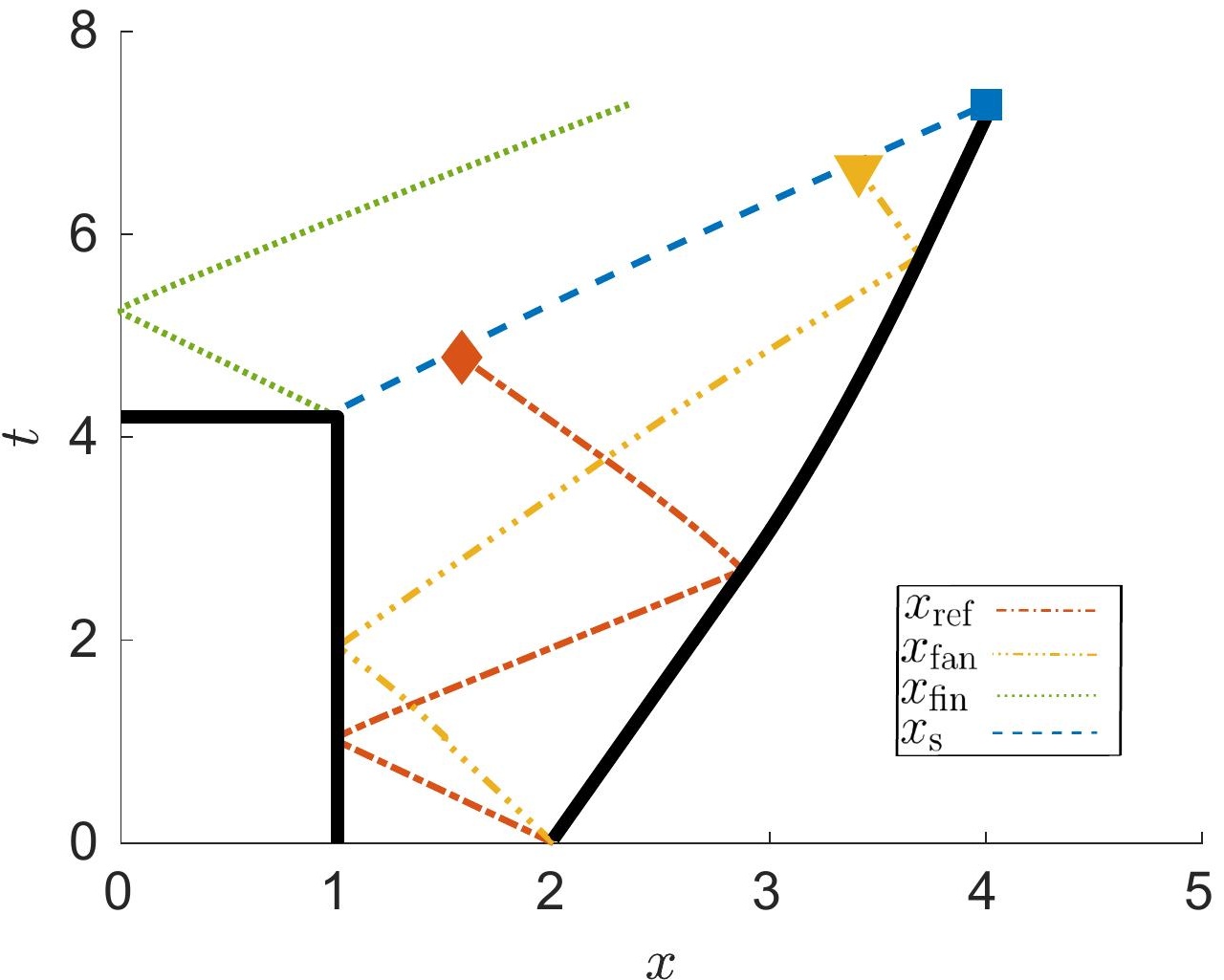




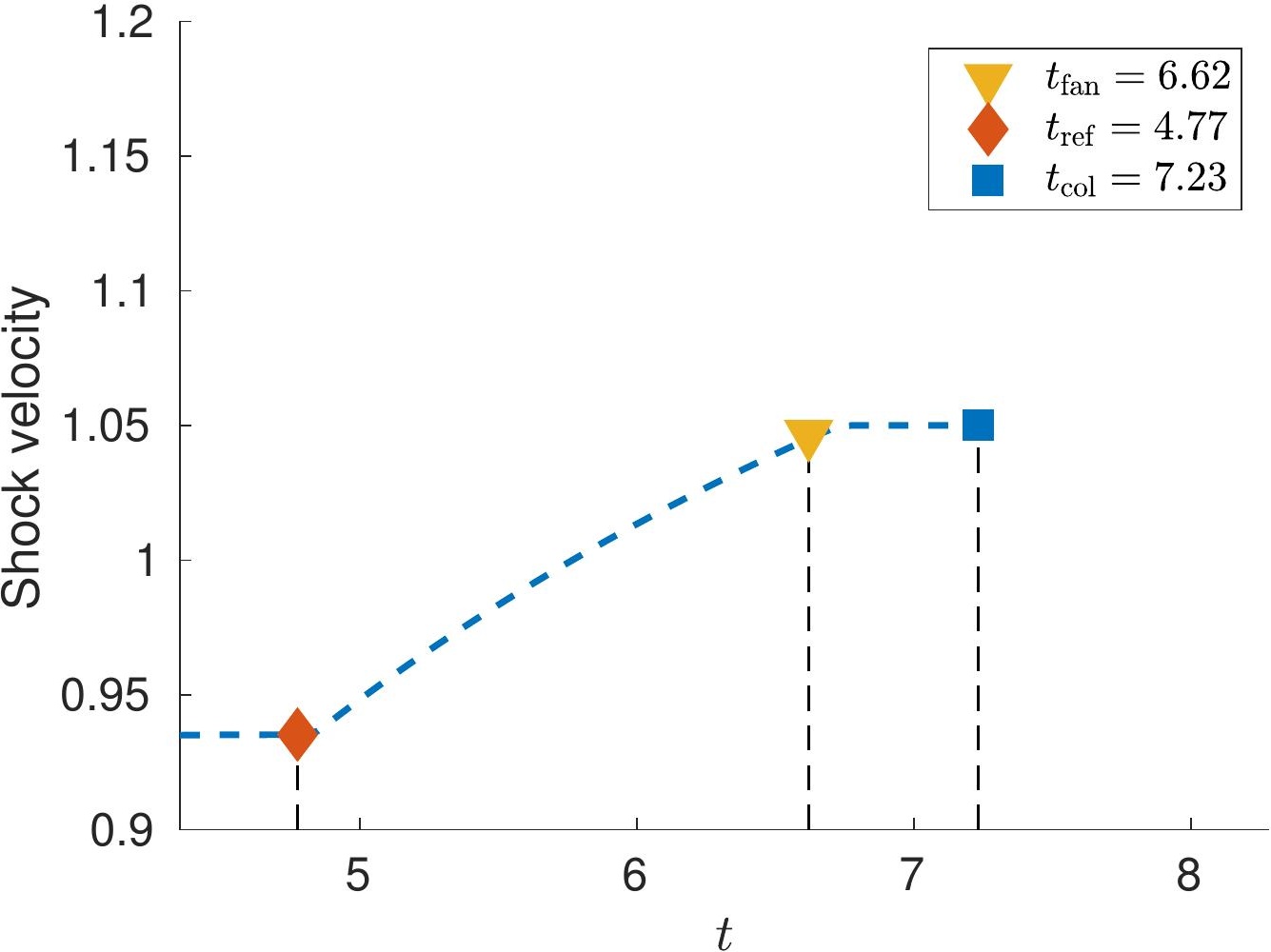




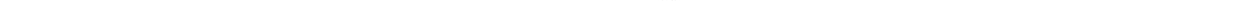




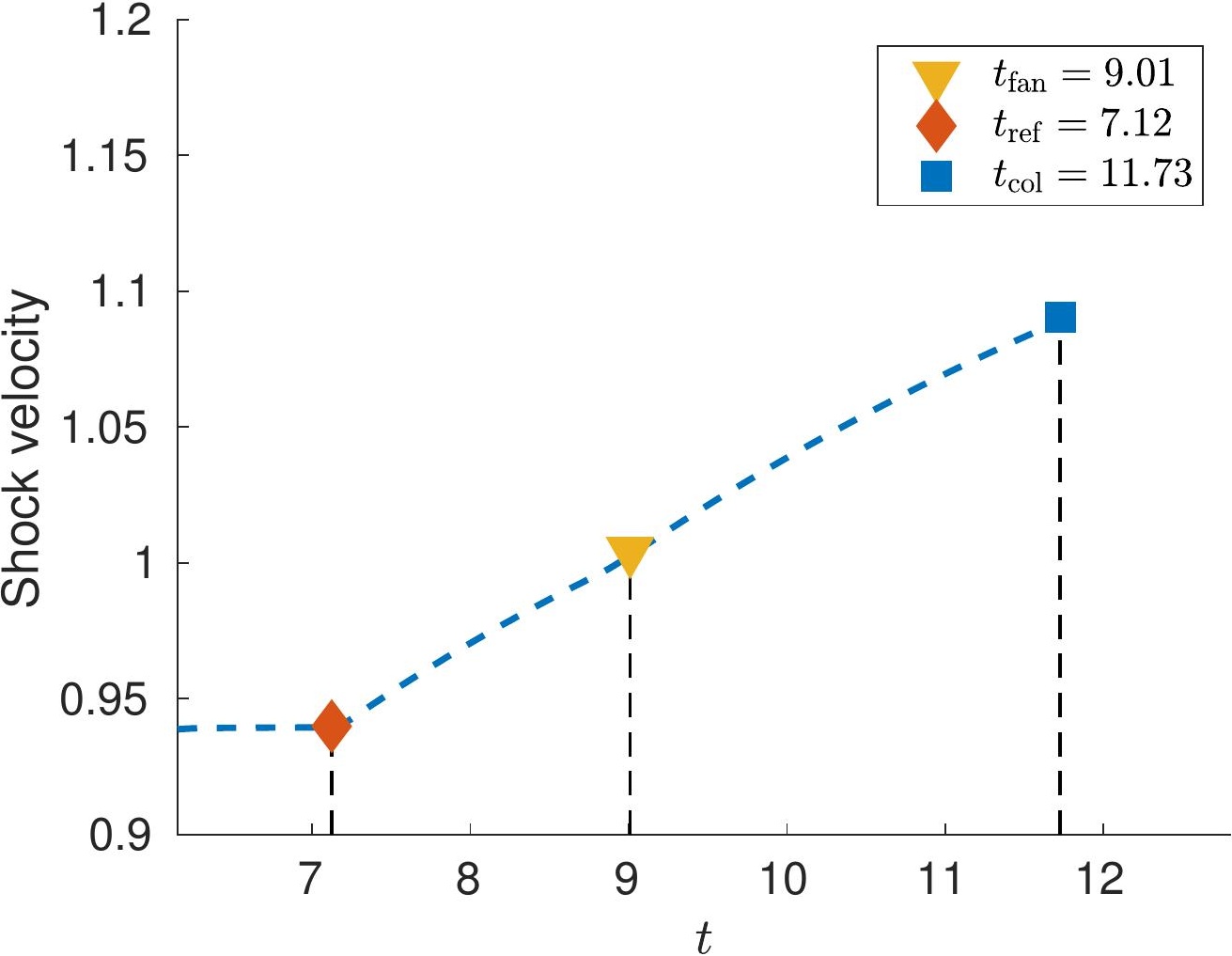




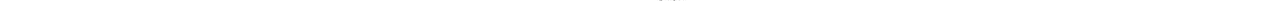









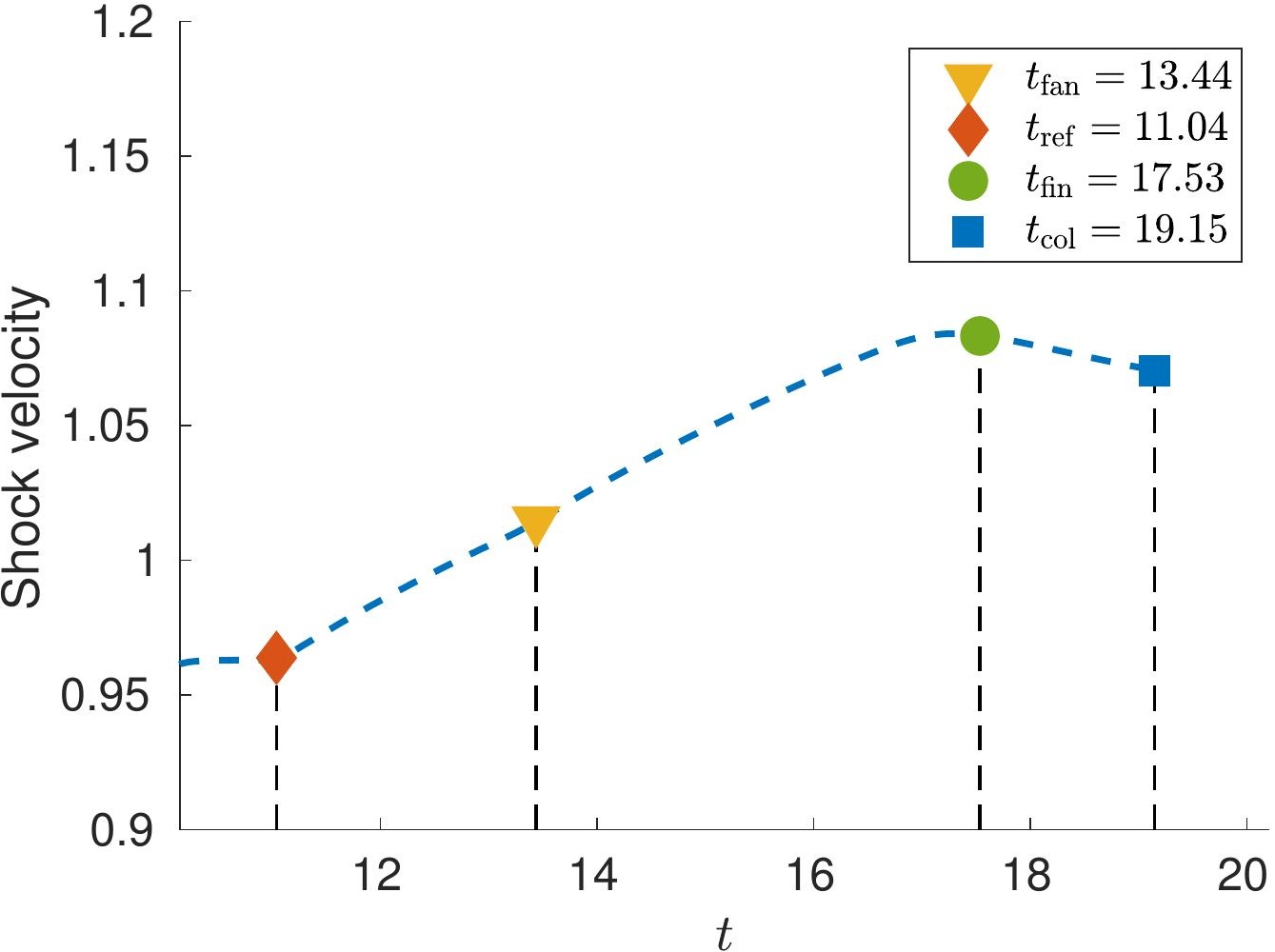




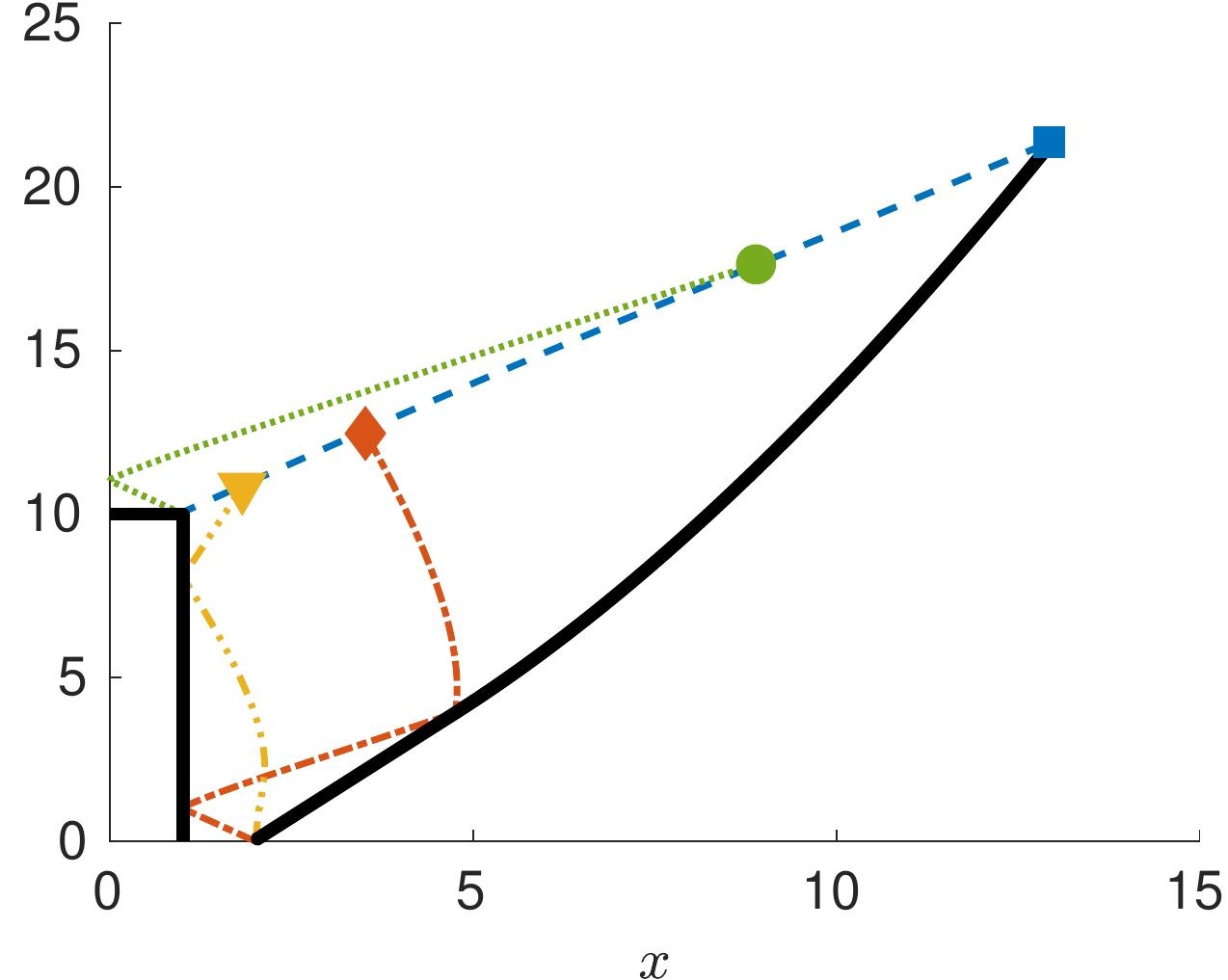




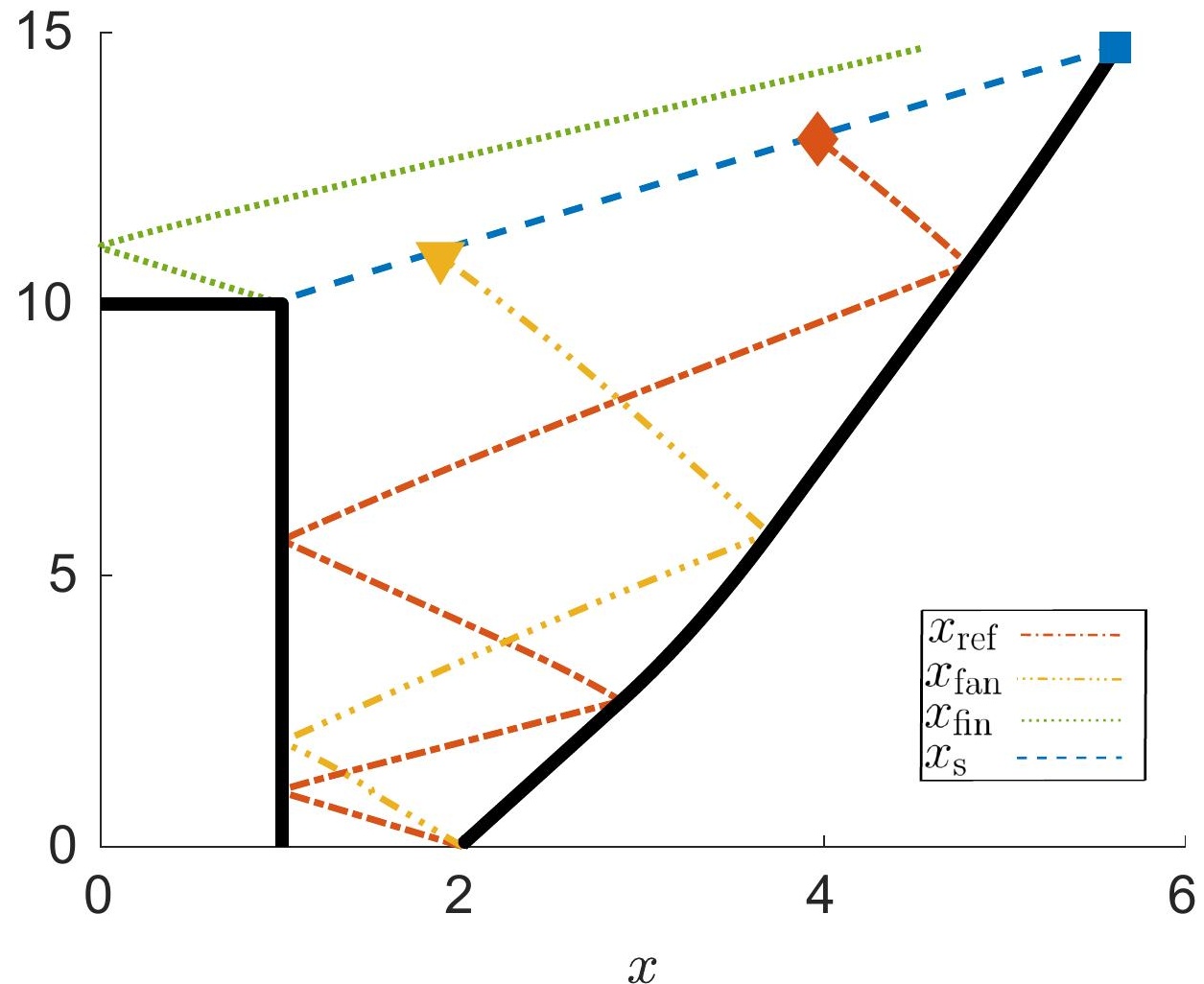




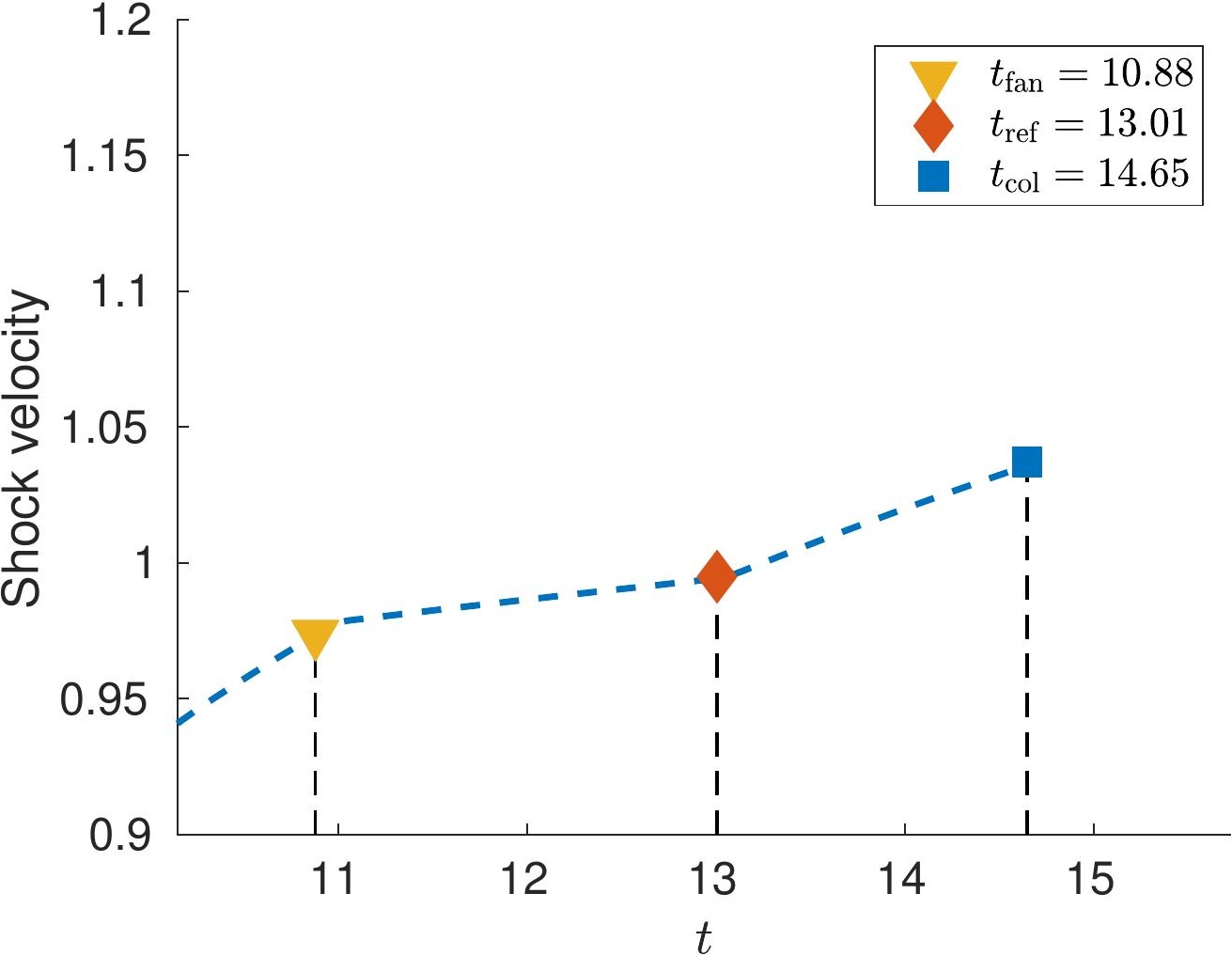









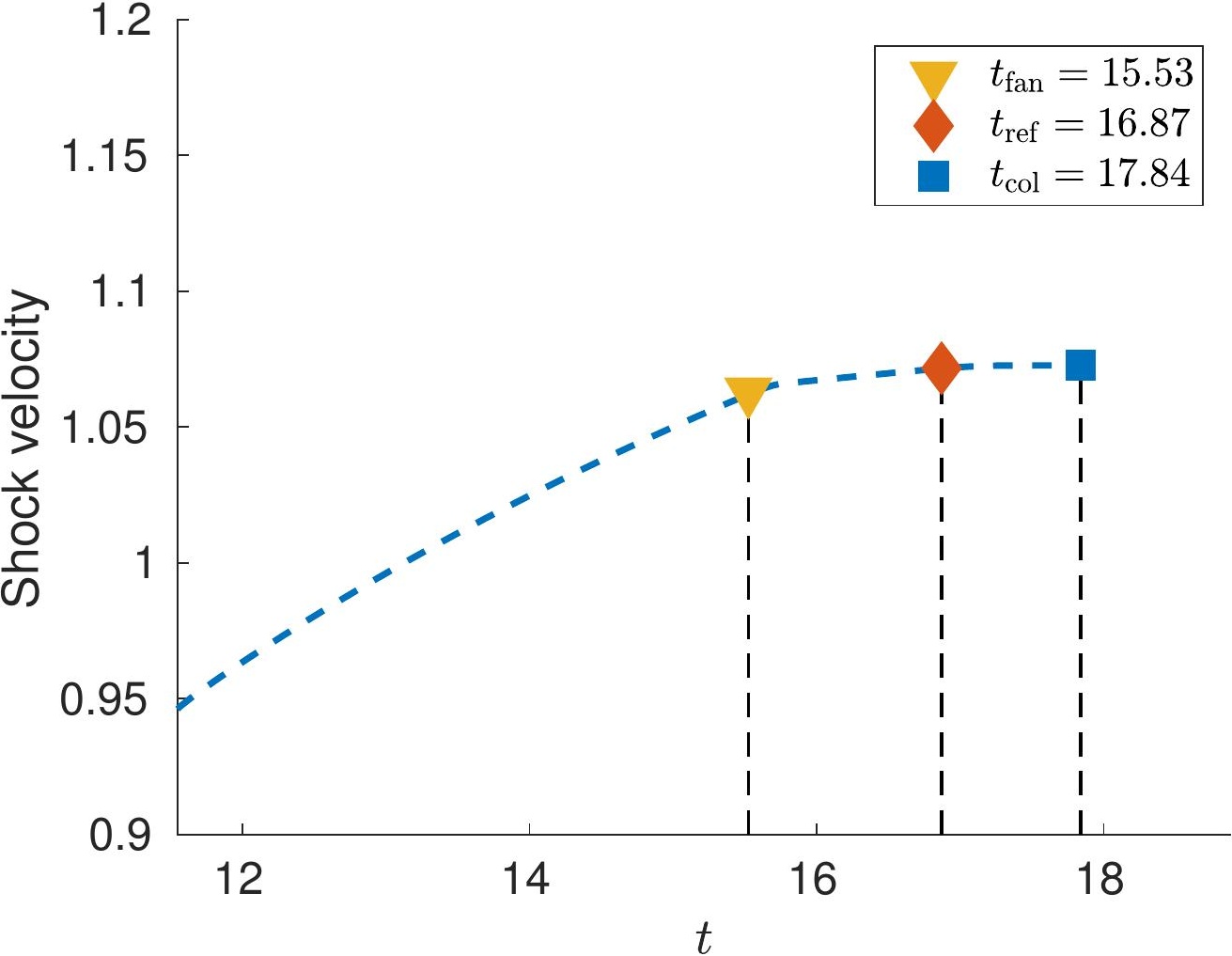









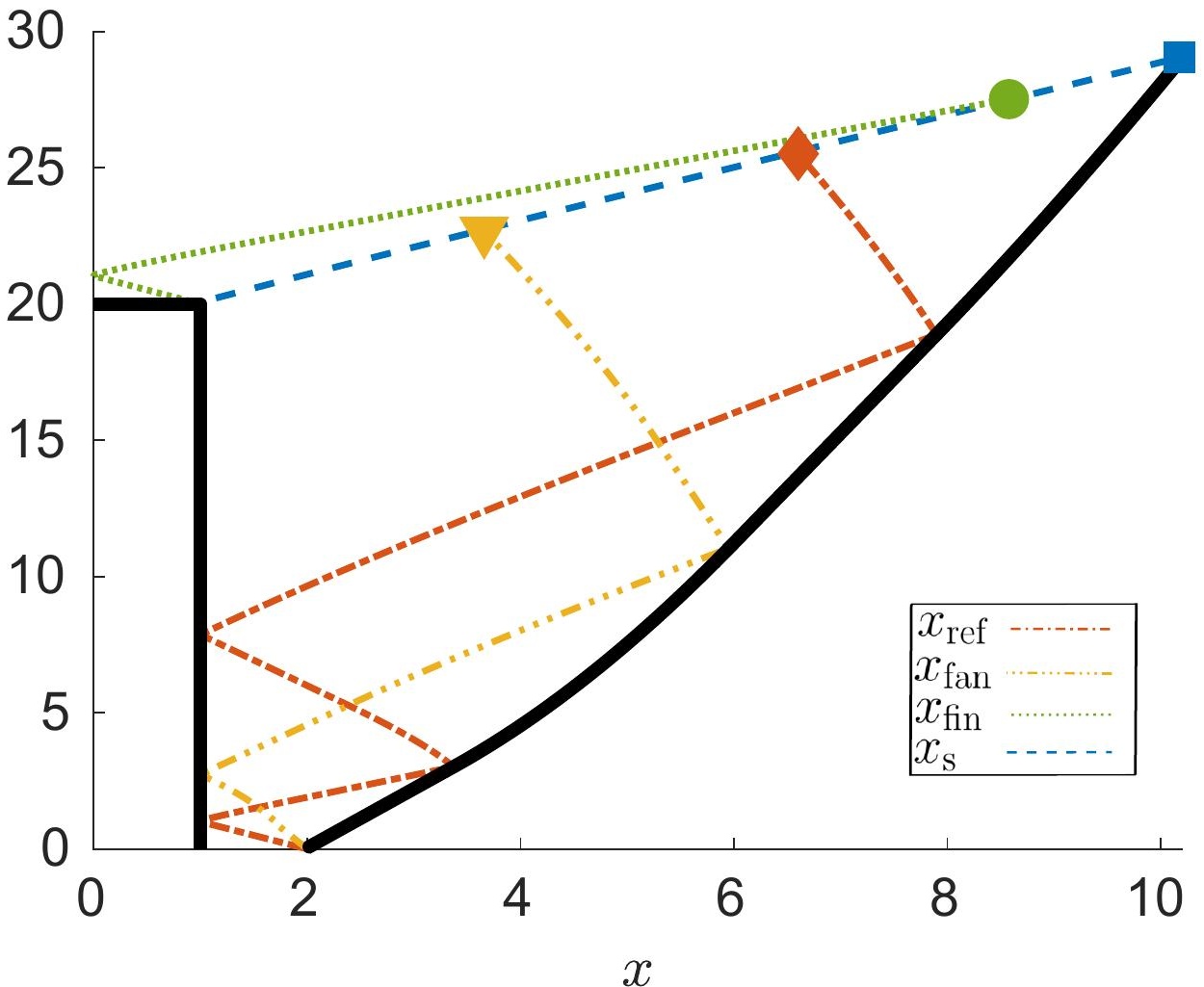




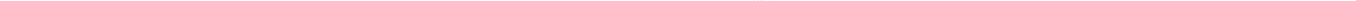




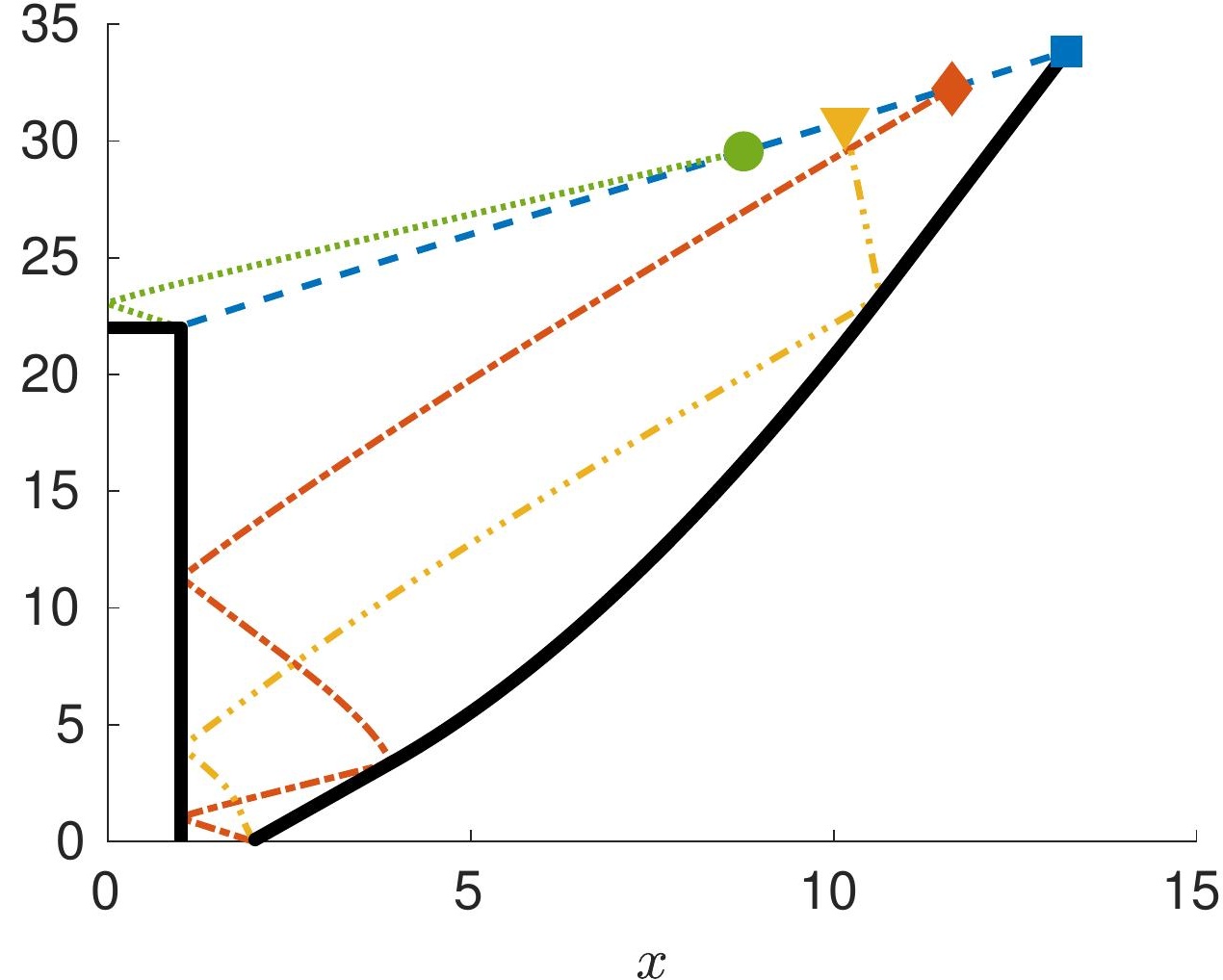














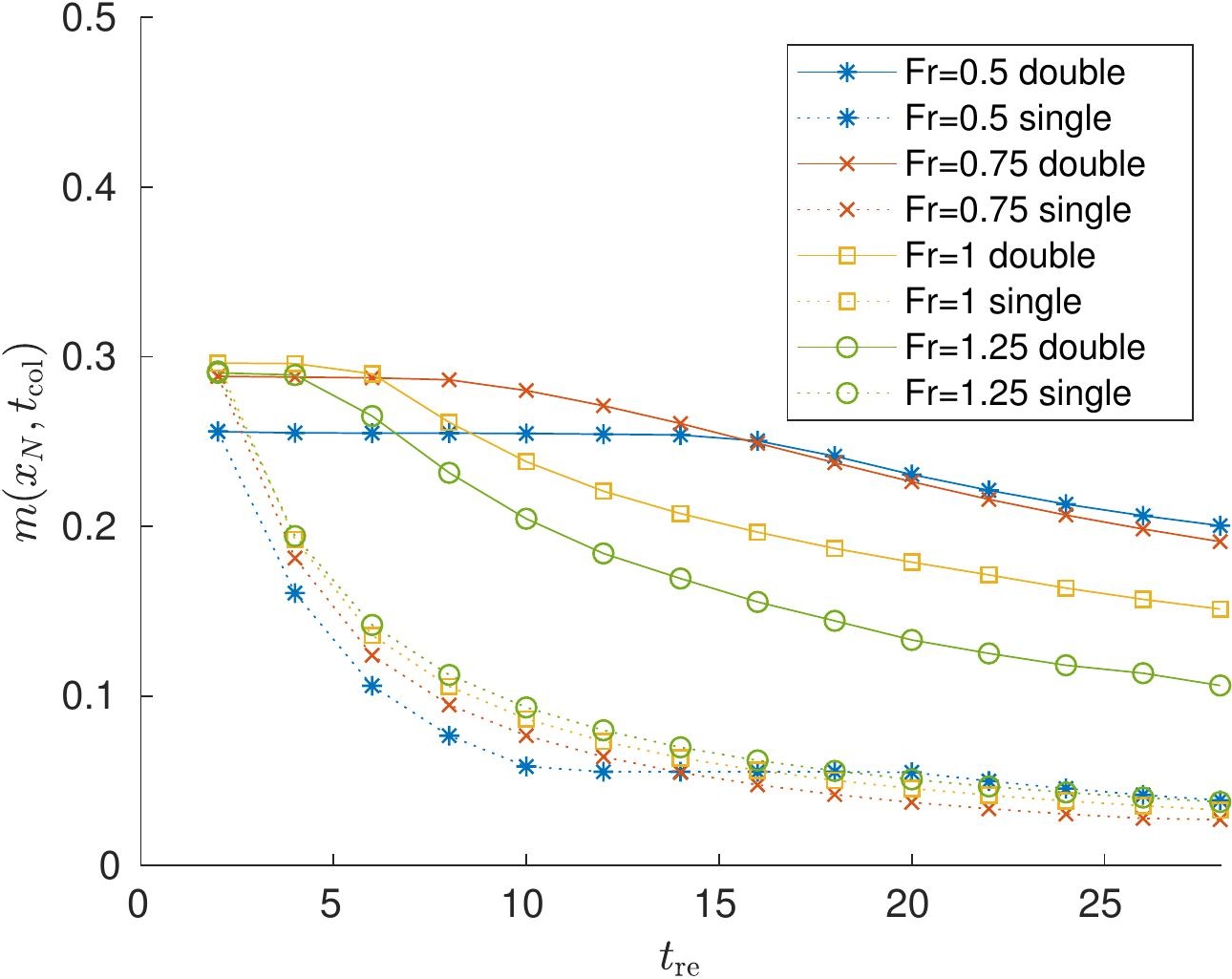




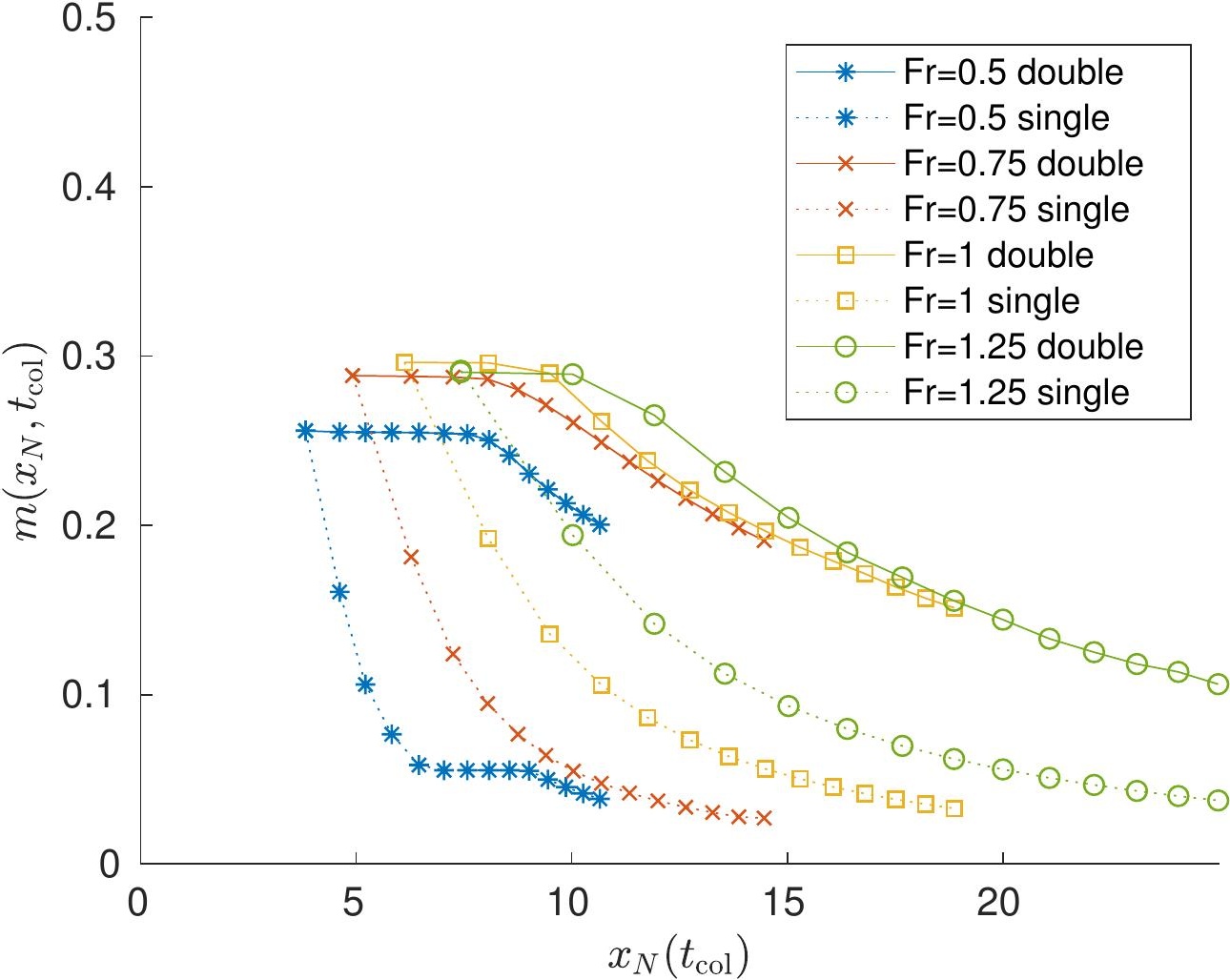




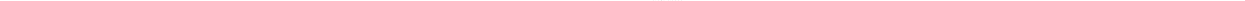




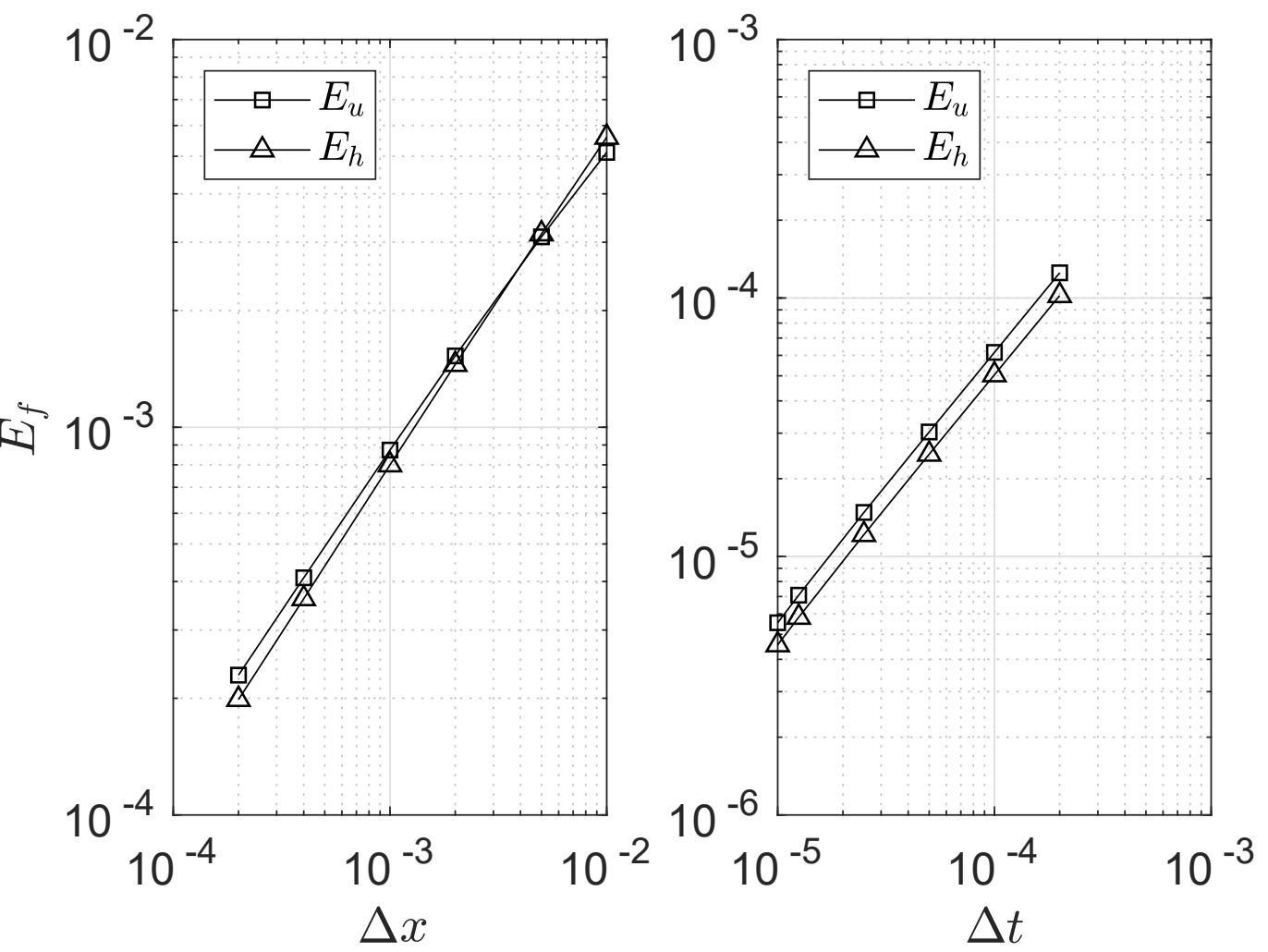




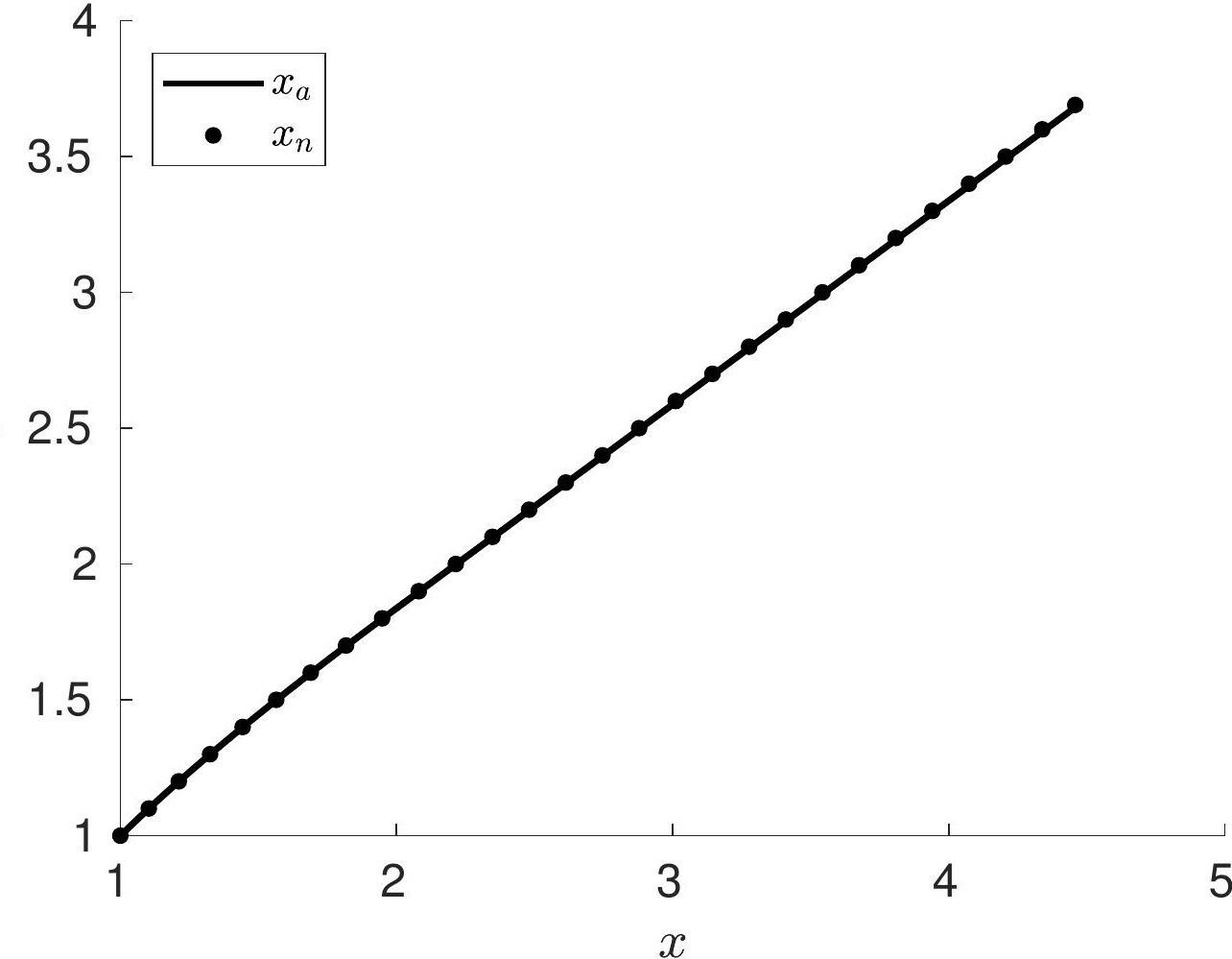

